\title{
The Gambia: Request for a Three-Year Arrangement Under the Poverty Reduction and Growth Facility and for Additional Interim Assistance Under the Enhanced Initiative for Heavily Indebted Poor Countries-Staff Report; Press Release on the Executive Board Discussion; and Statement by the Executive Director for The Gambia
}

In the context of the request for a three-year arrangement under the Poverty Reduction and Growth Facility and for additional interim assistance under the enhanced Initiative for Heavily Indebted Poor Countries, the following documents have been released and are included in this package:

- the staff report for the Request for a Three-Year Arrangement Under the Poverty Reduction and Growth Facility and for Additional Interim Assistance Under the Enhanced Initiative for Heavily Indebted Poor Countries, prepared by a staff team of the IMF, following discussions that ended on November 16, 2006, with the officials of The Gambia on economic developments and policies. Based on information available at the time of these discussions, the staff report was completed on February 7, 2007. The views expressed in the staff report are those of the staff team and do not necessarily reflect the views of the Executive Board of the IMF;

- $\quad$ a Press Release summarizing the views of the Executive Board as expressed during its February 21, 2007 discussion of the staff report that completed the request; and

- $\quad$ a statement by the Executive Director for The Gambia.

The documents listed below have been or will be separately released.

Letter of Intent sent to the IMF by the authorities of The Gambia*

Memorandum of Economic and Financial Policies by the authorities of The Gambia*

Technical Memorandum of Understanding*

*Also included in Staff Report

The policy of publication of staff reports and other documents allows for the deletion of market-sensitive information.

To assist the IMF in evaluating the publication policy, reader comments are invited and may be sent by e-mail to publicationpolicy@imf.org.

Copies of this report are available to the public from

International Monetary Fund • Publication Services

$70019^{\text {th }}$ Street, N.W. • Washington, D.C. 20431

Telephone: (202) 623-7430 • Telefax: (202) 623-7201

E-mail: publications@imf.org • Internet: http://www.imf.org

Price: $\$ 18.00$ a copy

International Monetary Fund

Washington, D.C. 

INTERNATIONAL MONETARY FUND

\title{
THE GAMBIA
}

\section{Request for a Three-Year Arrangement Under the Poverty Reduction and Growth Facility and for Additional Interim Assistance Under the Enhanced Initiative for Heavily Indebted Poor Countries}

\author{
Prepared by the African Department \\ (In consultation with other Departments) \\ Approved by Michael Nowak and Adrienne Cheasty \\ February 7, 2007
}

- $\quad$ Discussions on a new three-year Poverty Reduction and Growth Facility (PRGF) arrangement were held in Banjul during November 3-16, 2006. This followed the Gambian authorities' successful implementation of a staff-monitored program (SMP) over the period October 2005-March 2006. The last PRGF arrangement went off track soon after its approval in 2002 and expired in July 2005 without completion of a single review.

- $\quad$ The Gambia reached the decision point under the Heavily Indebted Poor Countries (HIPC) Initiative in December 2000, but is yet to reach the completion point. One outstanding prerequisite is satisfactory implementation of a PRGF-supported program for at least six months and the completion of at least one review. The Gambia will be eligible for assistance under the Multilateral Debt Relief Initiative (MDRI) once it reaches the HIPC completion point.

- The authorities are requesting a PRGF arrangement in the amount of SDR 14 million (45 percent of quota) and additional HIPC interim assistance in the amount of SDR 0.36 million. The Gambia's outstanding purchases and loans from the Fund at endDecember 2006 amounted to SDR 11.8 million (38 percent of quota).

- $\quad$ The mission met, among others, President Jammeh, Mr. Bala Gaye (Secretary of State for Finance and Economic Affairs), Mr. Jatta (Governor of the Central Bank of The Gambia), members of the Public Accounts Committee of the national assembly, and representatives of civil society organizations and the donor community.

- $\quad$ The mission team comprised Messrs. Tsikata (head), Srour, Sriram (all AFR), Marsh (PDR), and Segura-Ubiergo (Resident Representative to The Gambia and Senegal). Mr. Goodwin and Ms. Grochalska from FIN joined the mission to conduct an update safeguards assessment of the Central Bank of The Gambia. Ms. Lephoto (OED) and Mr. Soh (World Bank) participated in discussions with the authorities.

- $\quad$ President Jammeh was re-elected to a third five-year term in September 2006, with 67 percent of the votes cast. International observers described the election as fair. 


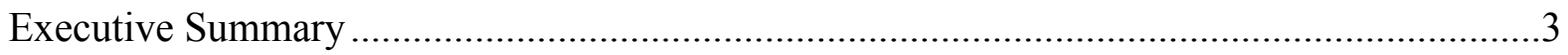

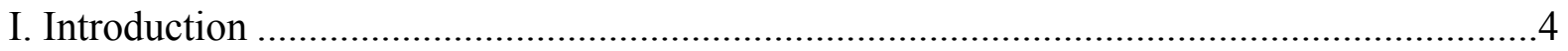

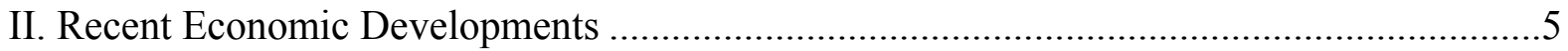

III. Macroeconomic Framework and Policies...............................................................

A. Consolidating Macroeconomic Stability......................................................

B. Strengthening Public Financial Management and Accountability .........................11

C. Strengthening Central Bank of The Gambia Internal Controls and

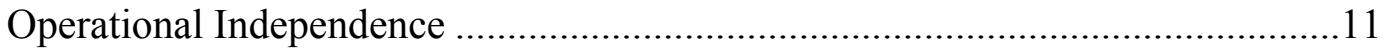

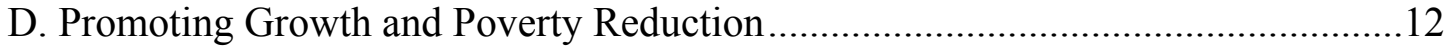

E. Toward HIPC Completion Point ..................................................................... 13

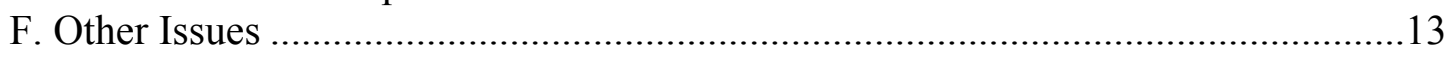

IV. Access Level and Financing Assurances...............................................................13

V. Prior Actions, Program Monitoring, and Capacity to Repay the Fund............................14

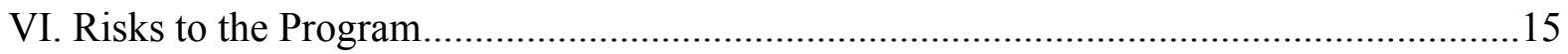

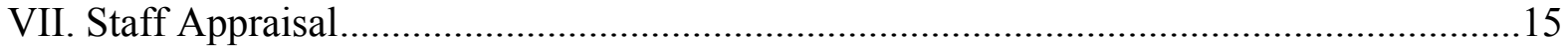

Tables

1. Selected Economic and Financial Indicators, 2004-11 ............................................17

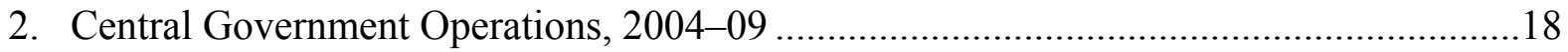

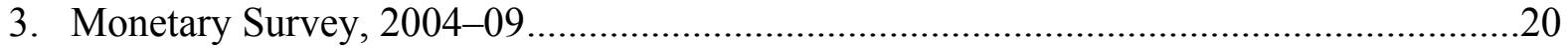

4. Analytical Account of the Central Bank of The Gambia, 2004-09...............................21

5. Balance of Payments, 2004-11 ...................................................................................22

6. Financial Soundness Indicators for the Banking Sector, 2000-September 2006 .............23

7. Heavily Indebted Poor Countries Initiative Completion Point Triggers .........................24

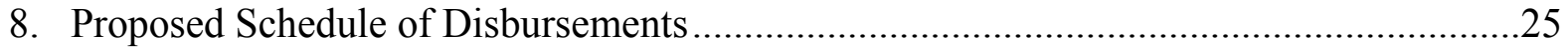

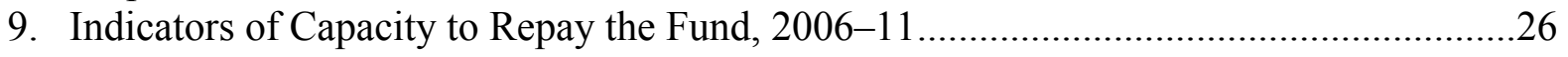

Appendix

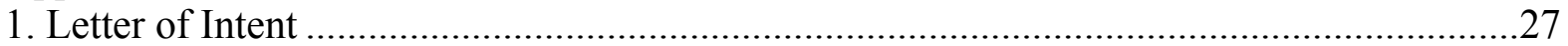

Attachment I. Memorandum of Economic and Financial Policies ........................29

Attachment II. Technical Memorandum of Understanding ..................................39 


\section{EXECUTIVE SUMMARY}

The Gambian authorities are requesting a three year PRGF arrangement to support their economic reform program and to help them make progress toward HIPC completion point. The program aims to consolidate recently achieved macroeconomic stabilization while also addressing the formidable challenges faced by the country, including debt distress, vulnerability to exogenous shocks, and widespread poverty.

\section{Recent developments}

- $\quad$ Real GDP growth recovered from a drought-induced decline in 2002 to average about 6 percent a year during 2003-06, outpacing population growth estimated at 2.8 percent a year.

- $\quad$ The authorities succeeded in reducing inflation from a peak of 21 percent per year in August 2003 to annual rates below 3 percent since June 2005.

- $\quad$ Fiscal performance in 2006 was stronger than in 2005, but not as strong as envisaged in the budget on account of expenditure overruns associated with an African Union summit held in Banjul in July. High domestic interest payments have been crowding out discretionary expenditures including PRSP-related spending.

- $\quad$ After contracting in 2004, credit to the private sector rebounded strongly in 2006.

- The economy has stayed relatively competitive in spite of a slight appreciation of the nominal and real effective exchange rate indices.

\section{Key elements of the PRGF-supported program}

Program objectives draw on the authorities' recently completed second PRSP. The medium-term (2007-09) assumptions and targets include (i) annual growth of 6-7 percent; (ii) annual inflation in the range of 2-4 percent; (iii) fiscal basic balance surpluses of about 3 percent of GDP a year to bring down domestic public debt to a sustainable path; and (iv) maintenance of international reserves at 4-5 months of imports. Based on indications from donors, the program projects a significant increase in net external financing which allows for increased growth-promoting and poverty-reducing government spending.

Fiscal adjustment, which is central to the program, is used to support a reduction in domestic real interest rates. Structural reforms include measures to (i) enhance internal controls and operational independence of the central bank to underpin macroeconomic stability; (ii) strengthen public financial management and accountability to ensure that public resources are used effectively and efficiently; and (iii) deepen financial intermediation.

Failure to maintain fiscal discipline poses the greatest risk to the program. President Jammeh assured staff of his government's commitment to successfully implement the program. If all goes well, completion of the first program review could be combined with reaching the HIPC completion point in July/August 2007. 


\section{INTRODUCTION}

1. The Gambian authorities are requesting a new three-year arrangement under the PRGF to support their economic reform program and to help them make progress toward completion point under the HIPC initiative. The authorities have been successful in stabilizing the economy over the last three years-inflation is down to low single-digit levels, the exchange rate has been relatively stable, and international reserves have been rebuilt to comfortable levels. Nevertheless, the country continues to face formidable economic challenges; it is debt-distressed, the economy remains vulnerable to exogenous shocks, and over 60 percent of the population lives below the poverty line.

2. The primary objectives of the program are to consolidate recent macroeconomic achievements and promote sustained high growth and poverty reduction. The program draws on the authorities' recently completed second Poverty Reduction Strategy Paper (PRSP II) which integrates the Millennium Development Goals (MDGs) into its objectives. PRSP II is to be implemented during 2007-11. Policies to promote growth include increased public investment in infrastructure and human capital, and measures to improve the investment climate. Poverty reducing measures include steps to ensure that budget execution is better aligned to PRSP II priorities than was the case with the first PRSP. ${ }^{1}$

3. Program design is also informed by the findings of the ex post assessment (EPA) of Fund-supported programs in The Gambia undertaken by Fund staff in $2005 .{ }^{2}$ The EPA concluded that programs had failed to achieve many of their objectives because of insufficient commitment to key reforms on the part of the authorities (reflecting a lack of ownership), as well as flaws in program design (e.g., over-optimistic revenue projections and inadequate attention to capacity constraints).

4. Conditions are propitious for implementing far-reaching economic reforms. The beginning of a new five-year term for President Jammeh, the unveiling of an MDG-based PRSP II, and indications of increased project aid from development partners, provide an opportunity for the country to make a decisive break from past stop-go policies, remove structural impediments to private investment, and improve public financial management and accountability.

\footnotetext{
${ }^{1}$ Implementation of the first PRSP which covered the period 2002-05 was uneven (MEFP, paragraph 4).

${ }^{2}$ IMF Country Report No. 06/11 (January 2006).
} 


\section{RECENT ECONOMIC DEVELOPMENTS}

\section{Real GDP growth recovered from} a drought-induced decline in 2002 to average about 6 percent annually during 2003-06, outpacing estimated 2.8 percent annual population growth.

The fastest growing sectors were hotels and restaurants (reflecting increased tourist arrivals), construction, and telecommunications. Services - mainly tourism-related activities, and transport and communications - dominate the economy, contributing over 60 percent of GDP.

\section{A marked slowdown in broad} money growth helped lower year-onyear consumer inflation from a peak of 21 percent in August 2003 to less than 3 percent since June 2005. As the macroeconomic environment improved, the $\mathrm{CBG}$ eased its monetary policy stance by gradually lowering the rediscount rate from 34 percent in September 2004 to 14 percent in October 2006. It also reduced the required reserve ratio on commercial banks' deposit liabilities from 18 percent to 16 percent in October 2006.

\section{After a strong adjustment effort} in 2004, the fiscal basic balance deteriorated in $\mathbf{2 0 0 5}$, reflecting the impact of extrabudgetary spending, lower customs revenues, and higher domestic interest payments. ${ }^{3}$

Preliminary indications suggest that fiscal performance improved in 2006,
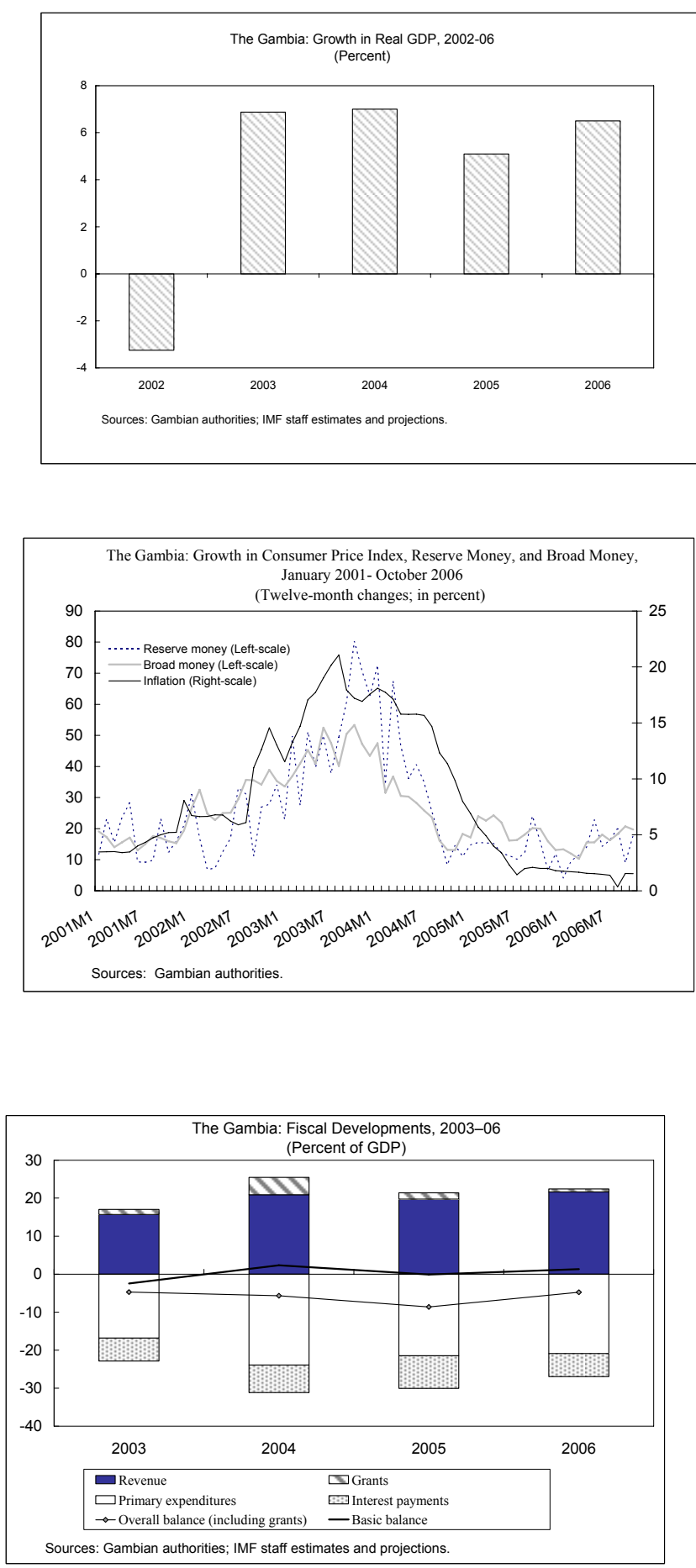

\footnotetext{
3 The basic balance is defined as revenues (excluding grants) minus total expenditure and net lending, excluding externally financed capital spending.
} 
though not as much as envisaged in the budget. Revenues were broadly in line with budget projections, while expenditures exceeded budgeted levels mainly on account of overruns associated with hosting the African Union (AU) summit. Interest payments - 80 percent of which are on domestic debt-fell as envisaged to 6.6 percent of GDP, but remained some 35 percent of current expenditures. The larger-than-expected deficit was financed by increased domestic borrowing and additional capital revenue. The stock of outstanding domestic public debt is estimated at 32 percent of GDP at end-2006. In addition, domestic payments arrears accumulated over several years are estimated at 3 percent of GDP.

8. Nominal interest rates have fallen, but real rates remain very high. Notably, reductions in lending rates have lagged behind other key rates, reflecting a high-risk lending environment and insufficient competition among banks.

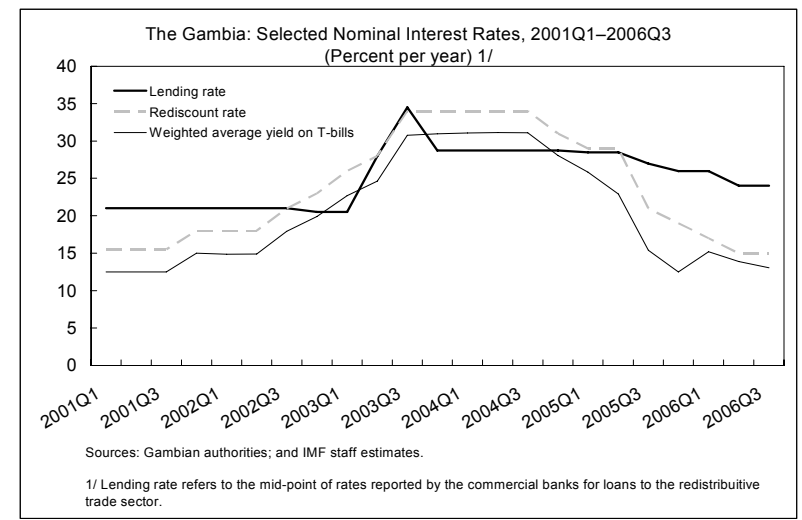

9. Credit to the private sector and public enterprises has rebounded strongly after contracting in 2004. Falling yields have reduced the attractiveness of treasury bills to banks relative to lending. Outstanding claims increased by 30 percent in the twelve months ending in September 2006, with loans to the agriculture, construction, and tourism sectors registering the fastest growth rates.
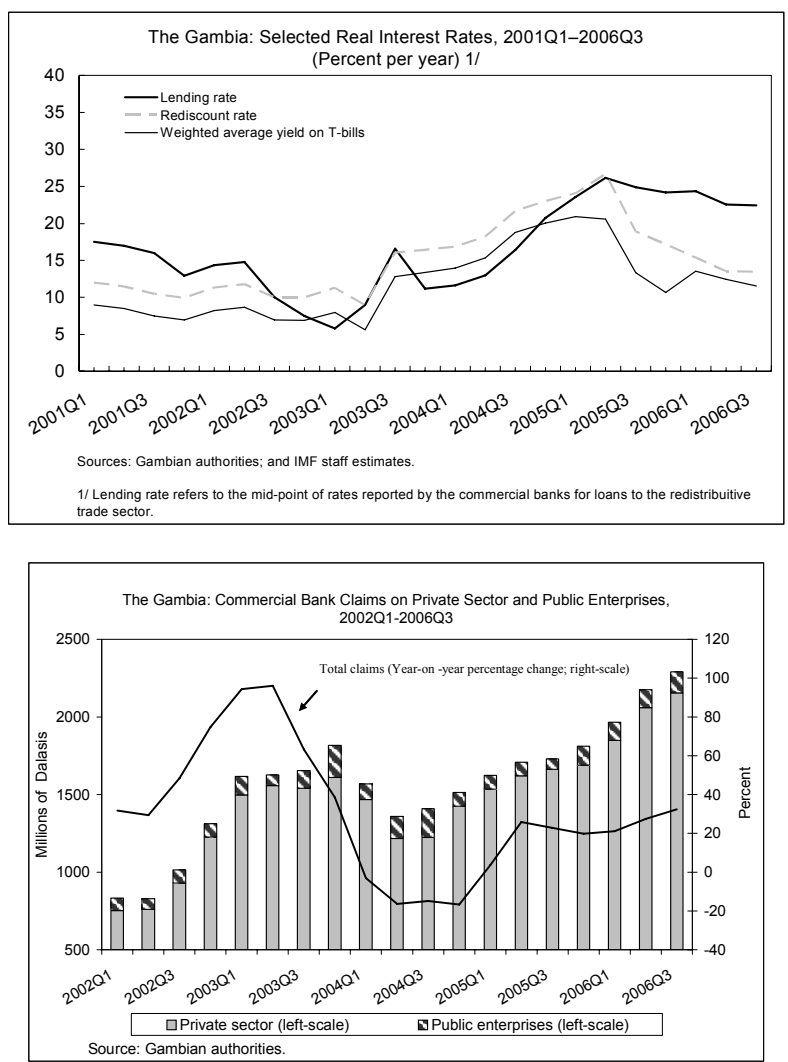

\section{After widening substantially in 2005, the external current account deficit} (including official transfers) narrowed significantly in 2006 (Table 1). However, at 14 percent of GDP, the current account deficit remains large. Highlights of external sector performance in 2006 included strong growth in tourism earnings and a recovery in groundnut exports from a near collapse in 2005. Foreign direct investment (FDI) and official concessional loans have been the main sources of financing for the current account deficit. 
The level of gross international reserves remains comfortable, at slightly more than four months of imports.

11. The dalasi has appreciated slightly in nominal and real effective terms over the last two years, after massive depreciations during 2002-03. The recent appreciation reflects increased inflows of remittances, transfers, and FDI. The economy remains relatively competitive, as demonstrated by robust growth in tourism receipts (the main source of foreign exchange earnings).

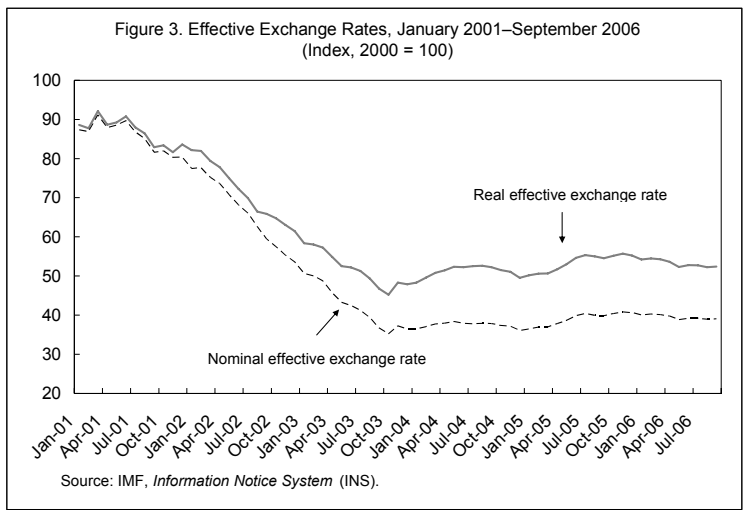

12. The Gambia has a de facto managed floating exchange rate system. The CBG's intervention in the foreign exchange market in the last two years has been largely limited to purchases to augment its international reserves.
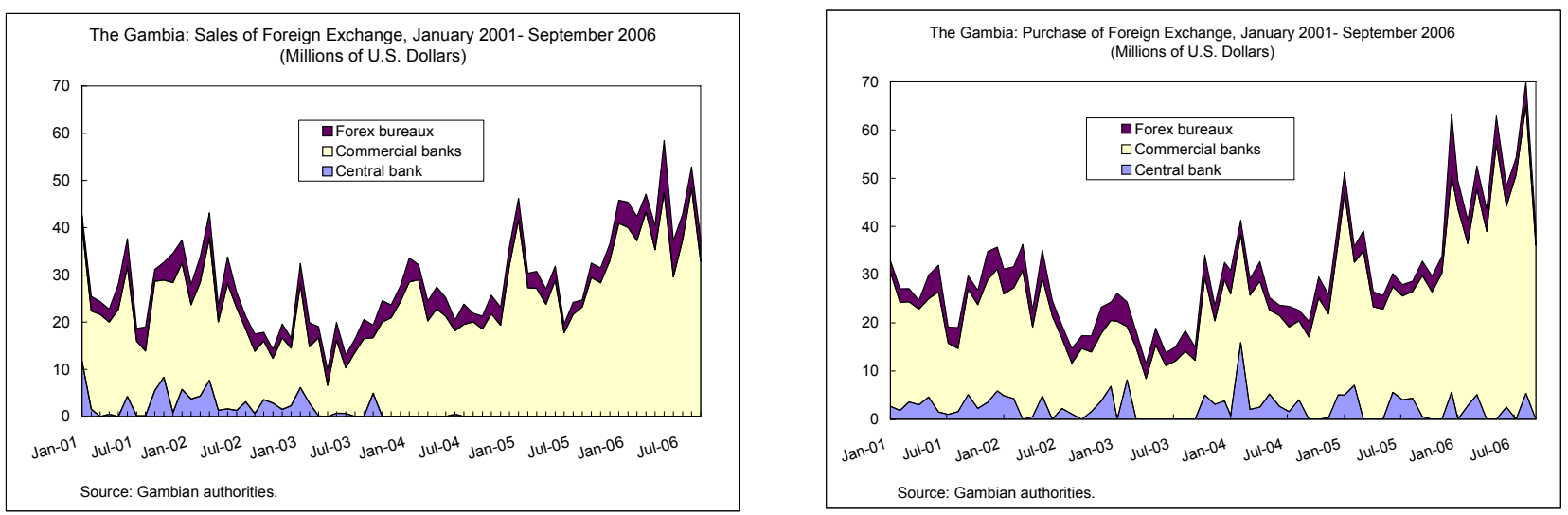

\section{MACROECONOMIC FrameWork AND Policies}

13. The most pressing macroeconomic challenge facing the authorities is how to rebalance the policy mix in order to lower real interest rates, stimulate private investment, and create fiscal space for growth-promoting and poverty-reducing government expenditures. Fiscal adjustment is central to the program, designed to support a reduction in domestic interest rates. Increased external aid will help avoid undue fiscal contraction and boost growth. Structural reforms under the program include: measures to enhance CBG internal controls and operational independence to sustain macroeconomic stability; a strengthening of public financial management to ensure that aid and domestic resources are used effectively in line with national priorities; and creation of a credit reference bureau to deepen financial intermediation. 


\section{A. Consolidating Macroeconomic Stability}

14. Key assumptions and objectives underlying the program's medium-term macroeconomic framework for 2007-09 are (Tables 1-5): ${ }^{4}$

- $\quad$ Real GDP average annual growth of 6.3 percent.

- $\quad$ Annual inflation in the range of 2-4 percent.

- $\quad$ The fiscal basic balance achieves an average annual surplus of 2.7 percent of GDP to bring down domestic public debt to a sustainable path, and to clear domestic payments arrears.

- $\quad$ The external current account deficit (including official transfers) narrows steadily from 14 percent of GDP in 2006 to 11 percent in 2009, reflecting strong growth in tourism earnings and a slowdown in import growth as the level of FDI and official loans taper off.

- International reserves are maintained around the equivalent of 4-5 months of import cover.

15. The services and industry sectors are expected to lead growth. Growth rates were revised, compared to those presented in the 2006 Article IV staff report, based on revised official national accounts estimates for 2004-06 and indications of sustained high levels of externally financed investments in the country. FDI in infrastructure related to tourism services has Structure of Real GDP and Sources of Growth, 2001-09 ${ }^{1}$ begun to pay off as tourist arrivals have risen sharply. The industry sector (especially construction) is projected to sustain the robust average annual growth rate (7 percent) of the last six years. Agriculture is projected to grow at 1 percent higher than the average over the last six years, but significantly less than the average for the last three years. Some FDI

\begin{tabular}{lrccc}
\hline & & \multicolumn{3}{c}{ Average Annual Growth Rates } \\
\cline { 3 - 5 } \multicolumn{1}{c}{ Sector } & Share in & $\begin{array}{c}2001-06 \\
\text { Est. }\end{array}$ & $\begin{array}{c}\text { 2004-06 } \\
\text { Est. }\end{array}$ & $\begin{array}{c}\text { 2007-09 } \\
\text { Proj. }\end{array}$ \\
\hline Agriculture & 2006 & 3.9 & 7.3 & 4.9 \\
Industry & 13.3 & 7.3 & 7.2 & 7.3 \\
Services & 63.5 & 5.3 & 5.4 & 6.4 \\
Real GDP & 100.0 & 4.9 & 6.0 & 6.2 \\
\hline
\end{tabular}

Sources: Gambian authorities; IMF staff estimates and projections.

${ }^{1}$ Based on real GDP at factor cost.

\footnotetext{
${ }^{4}$ The growth and inflation assumptions in this framework differ from those in PRSP II. They reflect discussions during the mission which took place after PRSP II had been completed. PRSP II assumes annual growth rates of 4.5 percent (much lower than recent average growth) and inflation of 5 percent.
} 
has gone into non-traditional agriculture and should boost growth in the sector.

\section{Proposed policies and external debt relief are expected to help the country} achieve debt sustainability. The stock of domestic public debt falls from 32 percent of GDP in 2006 to 23 percent in 2009. HIPC and MDRI debt relief help lower the net present value of external debt-to-GDP ratio from 41 percent in 2006 to 27 percent in 2009, falling below the indicative sustainability threshold for The Gambia (30 percent). A recent debt sustainability analysis (DSA) undertaken by staffs of the Fund and the World Bank concluded that The Gambia would remain at a moderate risk of falling back into debt distress even after receiving debt relief under the HIPC and MDRI initiatives. ${ }^{5}$ It stressed the importance of relying more on nondebt creating flows (grants and FDI) rather than loans for The Gambia's public debt sustainability. The program builds in a shift in external financing from loans to grants (paragraph 18), and new external borrowing will be contracted on concessional terms only.

\section{Fiscal policy}

\section{Fiscal adjustment is premised on sustaining revenues at about 21 percent of} GDP, falling domestic interest payments, and nonrecurrence of exceptional expenditures such as those related to the AU summit and presidential elections.

The fiscal effort is slightly higher in 2007 than in subsequent years to allow for a large repayment of arrears that year. Maintaining fiscal discipline is critical for containing the government's domestic borrowing requirement and putting downward pressure on interest rates to reduce the heavy burden of domestic debt service on the budget. The projected decline in domestic interest payments assumes a steady fall in the average real yield on treasury bills from about 13 percent in 2006 to 8 percent in 2009 . At end-2006, the average treasury bill yield across maturities had already fallen to The Gambia: Summary of Central Government Operations, 2003-09 (Percent of GDP)

\begin{tabular}{|c|c|c|c|c|c|}
\hline & $\begin{array}{r}2005 \\
\text { Actual } \\
\end{array}$ & $\begin{array}{l}2006 \\
\text { Prel. }\end{array}$ & $\begin{array}{l}2007 \\
\text { Proj. }\end{array}$ & $\begin{array}{c}2008 \\
\text { Proj. }\end{array}$ & $\begin{array}{r}2009 \\
\text { Proj. }\end{array}$ \\
\hline Revenue & 19.7 & 21.6 & 21.3 & 21.3 & 21.2 \\
\hline Grants & 1.7 & 0.8 & 8.6 & 5.0 & 4.5 \\
\hline Expenditure & 30.0 & 27.1 & 28.4 & 27.4 & 26.5 \\
\hline Current expenditure & 18.4 & 18.9 & 15.5 & 15.2 & 15.0 \\
\hline Of which: interest payments & 8.6 & 6.5 & 5.3 & 4.2 & 3.4 \\
\hline Capital expenditure & 11.0 & 7.7 & 12.0 & 11.2 & 10.5 \\
\hline Of which: externally financed & 10.2 & 6.8 & 10.2 & 8.5 & 7.8 \\
\hline Basic primary balance & 8.5 & 7.9 & 8.3 & 6.6 & 5.9 \\
\hline Basic balance $^{1}$ & -0.1 & 1.3 & 3.1 & 2.4 & 2.6 \\
\hline Overall balance & -8.6 & -4.7 & 1.4 & -1.1 & -0.7 \\
\hline Statistical discrepancy & -0.5 & 0.2 & 0.0 & 0.0 & 0.0 \\
\hline Financing & 9.2 & 4.5 & -1.4 & 1.1 & 0.7 \\
\hline External & 5.5 & 2.2 & 1.1 & 2.6 & 1.5 \\
\hline Domestic & 3.7 & 2.4 & -2.5 & -1.5 & -0.8 \\
\hline Of which: net borrowing & 3.3 & 2.6 & -0.6 & -1.0 & -0.8 \\
\hline \multicolumn{6}{|l|}{ Memorandum items: } \\
\hline Gross domestic debt & 35.5 & 31.6 & 30.3 & 26.4 & 23.1 \\
\hline Stock of domestic arrears & 4.5 & 2.9 & 0.5 & 0.0 & 0.0 \\
\hline
\end{tabular}

Sources: Gambian authorities; IMF staff estimates and projections.

${ }^{1}$ Domestic revenue minus expenditure and net lending, excluding externally financed capital expenditures. about 10 percent in real terms.

\footnotetext{
${ }^{5}$ See Appendix I of IMF Country Report No. 06/444 (December 2006).
} 
18. The program projects a significant increase in net external financing in US dollar terms, allowing for increased growth-promoting and poverty-reducing government expenditures. Project-related grants increase significantly in 2007 and then taper off. This reflects donor commitments to finance new projects, including a large

The Gambia: Net Official External Financing, 2003-09

(Millions of U.S. dollars, unless otherwise indicated) ${ }^{1}$

\begin{tabular}{|c|c|c|c|c|c|c|c|}
\hline & 2003 & 2004 & 2005 & 2006 & 2007 & 2008 & 2009 \\
\hline & & & & Prel. & Proj. & Proj. & Proj. \\
\hline Net official external financing & 24.8 & 41.2 & 33.2 & 14.1 & 39.7 & 45.9 & 40.0 \\
\hline Project grants & 4.6 & 13.2 & 7.1 & 3.8 & 32.0 & 29.0 & 28.9 \\
\hline HIPC grants (multilateral) & 4.0 & 5.0 & 0.6 & 0.0 & 1.7 & 1.2 & 0.9 \\
\hline Loan disbursements & 26.5 & 37.3 & 39.8 & 30.7 & 22.0 & 22.6 & 22.6 \\
\hline Amortization (-) & -10.3 & -14.4 & -14.4 & -20.5 & -19.0 & -12.5 & -17.8 \\
\hline Other debt relief (bilateral) & 0.0 & 0.0 & 0.0 & 0.0 & 2.9 & 5.6 & 5.4 \\
\hline \multicolumn{8}{|l|}{ Memorandum items: } \\
\hline Net official external transfers (percent of GDP) & 7.0 & 10.3 & 7.2 & 2.8 & 7.1 & 7.6 & 6.1 \\
\hline
\end{tabular}

Source: IMF staff estimates.

${ }^{1}$ Excluding technical assistance.

European Union-sponsored roads project, and commitments by the World Bank and the African Development Bank to shift the composition of their assistance from loans to grants. Staff projections of external financing represent about 60 percent of donor commitments, and are about half of the authorities' estimated requirement to fully implement PRSP II. The authorities are planning a donors conference in March/April 2007 where additional pledges will be sought.

\section{Monetary policy}

\section{To meet the program's inflation targets, broad money is targeted to expand at} slightly above the growth rate of nominal GDP. Falling real interest rates and strong capital and grants inflows should boost real money demand. Commercial bank lending rates are projected to fall, following recent monetary easing and greater competition in the banking system from the arrival of two new entrants in 2006 . The authorities need to monitor closely the capacity of the economy to absorb the expected increase in external resource inflows. Foreign-financed investment is likely to exert pressure on prices of scarce goods and services, such as skilled labor, while items such as food may not be significantly affected. The CBG considers it prudent to pause to assess the effects of the recent easing, before signaling any further change in policy. 


\section{B. Strengthening Public Financial Management and Accountability}

20. The authorities are committed to improving all aspects of the budget process (MEFP, paragraphs 18 and 19). Weaknesses in budget formulation combined with poor budget execution have in the past led to overspending and large domestic payments arrears. The launching of the World Bank supported Integrated Financial Management Information System (IFMIS) in January 2007, should improve budget management considerably, including more effective commitment control to avoid the incurrence of new arrears.

21. To enhance accountability in the use of public resources, the government intends to clear the backlog of unaudited government accounts in 2007 and improve the transparency and comprehensiveness of the accounts (MEFP, paragraphs 22 and 23). Accounts for 2000-03 have been submitted to the Auditor-General, and the government has undertaken to submit those for 2004-06 before the end of 2007. The government will set up a central project management and aid coordination directorate at the Department of State for Finance and Economic Affairs (DoSFEA) which will compile information on aid inflows and the uses to which they are put. The government will also collect quarterly financial information on key public enterprises to maintain a record of possible quasi-fiscal activities and help monitor potential risks to the budget.

22. The authorities are taking steps to enhance revenue administration and collection (MEFP, paragraph 17). The 2007 budget provides resources for the Gambia Revenue Authority to recruit additional staff in critical areas and to upgrade equipment and software. The forthcoming issuance of taxpayer identification numbers should also help to improve tax collection.

\section{Strengthening Central Bank of The Gambia Internal Controls and Operational Independence}

\section{The CBG is addressing vulnerabilities identified by a 2004 Safeguards}

Assessment, but is yet to make key control mechanisms fully effective. Notable progress has been made in strengthening accounting controls. However, vulnerabilities remain in controls over international reserves and procedures for extending credit to the government. The program contains measures to address the remaining vulnerabilities, based on the recent update Safeguards Assessment. Priority measures include semi-annual audits of monetary program data, the implementation of segregation of duties in the reserves management function, and a phased implementation of international accounting standards.

24. To strengthen the CBG's operational independence, the government has undertaken to recapitalize the CBG (MEFP, paragraph 26). A return to profitability in 2006 after many years of making losses should also strengthen the CBG (MEFP, paragraph 25). The authorities will ensure that by the end of 2007, government borrowing from the CBG is brought within the limit stipulated in the law (MEFP, paragraph 27). 


\section{Promoting Growth and Poverty Reduction}

\section{Promoting growth}

25. Tourism and trade are expected to remain important sources of growth. Tourism has been growing at an annual average rate of 10 percent since 2002, and should remain strong in light of recent and expected large investments. The impact on trade of a tariff increase in January 2006 as part of the implementation of the ECOWAS common external tariff needs further analysis. ${ }^{6}$ The authorities acknowledged that the tariff increase may adversely affect the re-export trade. However, they believe that the long-term benefits of duty-free access to the whole ECOWAS region would outweigh the costs. ${ }^{7}$

26. Weak transportation and energy infrastructure, the high cost of doing business, and a small domestic market remain serious impediments to growth. The authorities need to improve the investment climate, particularly in the legal and infrastructure areas. The Gambia was featured for the first time in the World Bank's “doing business" rankings in 2006. It performed well in relation to other sub-Saharan African countries $\left(14^{\text {th }}\right.$ out of 45 countries ranked) but did poorly globally, ranking $113^{\text {th }}$ out of 175 countries. Areas of greatest weakness were taxation of companies, protecting investors, and access to credit. The World Bank is assisting with several studies to guide improvements in the investment climate and to promote the expansion and diversification of exports (MEFP, paragraph 32).

\section{The CBG plans to establish a Credit Reference Bureau to help deepen financial} intermediation. The financial sector in The Gambia is relatively sound, with high profitability and liquidity ratios and a relatively low share of nonperforming loans in total outstanding loans (Table 6). However, loan-to-deposit ratios are low and spreads in commercial interest rates are very wide, reflecting a low level of competition in the banking system, and a high-risk environment (partly due to weaknesses in the legal system).

\section{Poverty reduction}

\section{Agriculture, which accounts for about one-quarter of GDP, is the principal} source of livelihood for the vast majority of the population. Groundnuts cultivation remains the mainstay of the agricultural sector (about one-third of value-added), although its share has been declining. Disruptions in groundnut marketing over the last few seasons adversely affected the income of farmers. Plans to privatize the Gambia Groundnut

\footnotetext{
6 The tariff rate on consumer goods, which form the bulk of reexports was increased from 18 percent to 20 percent. The sales tax on non-oil imports was also increased from 10 percent to 15 percent to align it with the domestic sales tax.

${ }^{7}$ Goods with a minimum of 35 percent value-added in the home country can be exported duty-free within ECOWAS.
} 
Corporation have stalled, and the World Bank is helping the authorities explore options for moving forward while safeguarding the welfare of farmers (MEFP, paragraph 33).

29. The authorities have undertaken to ensure that PRSP-related expenditures are well protected in budget execution. A distinction is made in the budget between PRSPrelated spending and discretionary spending at a departmental line-item level. Spending units have been instructed to ensure that allocations intended for poverty-reduction purposes are not diverted to other ends. The 2007 budget allocates over 40 percent of domesticallygenerated funds to poverty reduction (defined to include rehabilitation of key trunk roads).

\section{E. Toward HIPC Completion Point}

30. The HIPC completion point triggers are grouped under five headings (Table 7). They appear to have been met in two areas - poverty reduction and social sector reforms. In the area of macroeconomic stability, the conditions are expected to be met by end-July 2007 . Work to fulfill the conditions in the remaining two areas-governance and structural reforms - is in progress, and the authorities expect to satisfy all the conditions around mid2007.

\section{F. Other Issues}

31. Data weaknesses and low implementation capacity have hampered economic management in The Gambia. The authorities are receiving technical assistance from the World Bank and the Fund to improve the quality and timeliness of statistics on consumer prices, national accounts and balance of payments (MEFP, paragraph 35). The Fund has also been providing technical assistance in the areas of monetary statistics, monetary operations, banking supervision and public financial management.

\section{The authorities are initiating work on a comprehensive civil service reform} program. They have requested the World Bank to undertake a study that would guide the formulation and implementation of a plan to establish a more efficient and better remunerated civil service. The African Development Bank is also considering a project targeted at improving the capacity to implement the PRSP.

\section{AcCess LeVel and Financing Assurances}

33. The proposed access level is SDR 14 million (45 percent of quota) over three years (Table 8). This is in line with the norm for a fourth PRGF arrangement. ${ }^{8}$ The program projects small balance of payments surpluses over the medium-term, but experience suggests that adverse shocks can alter the outlook substantially. The recent DSA showed that the

${ }^{8}$ The Gambia's three previous ESAF/PRGF arrangements were approved in 1988, 1998, and 2002. 
external outlook is highly sensitive to assumptions about export growth and non-debt creating inflows.

34. The authorities are also requesting additional interim assistance under the enhanced HIPC Initiative in the amount of SDR 0.36 million in 2007. The Fund committed SDR 1.8 million in HIPC assistance at the decision point, of which SDR 80,000 was disbursed in interim assistance, before The Gambia's last PRGF-supported program went off-track.

35. The first year of the program (2007) is fully financed based on commitments from donors and expected debt relief from multilateral and bilateral creditors. It is assumed that most of the debt relief will come in the second half of 2007 after the country reaches HIPC completion point. The Fund has received financing assurances from the Paris Club. ${ }^{9}$ The bulk of The Gambia's external debt is owed to multilateral creditors, with the World Bank, African Development Bank and the Fund accounting for about 70 percent of total outstanding external debt at end-2005.

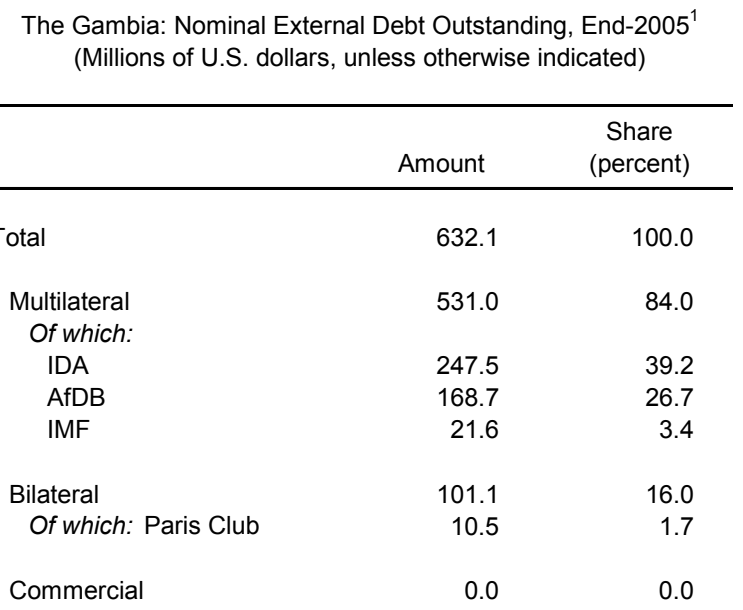

Source: Gambian authorities; IMF staff estimates.

${ }^{1}$ Data are not reconciled with creditor statements.

\section{Prior Actions, Program Monitoring, and Capacity to Repay the Fund}

36. The authorities undertook to implement the following actions prior to IMF Executive Board consideration of their request for the new PRGF arrangement:

(i) approval of a budget for 2007 by the National Assembly that is in line with the program with respect to the size of the fiscal basic balance; (ii) submission of government accounts for 2003 to the Auditor-General; and (iii) formulation of an action plan to bring government borrowing from the CBG in line with the limit under the CBG Act (2005) by end-2007.

37. Performance under the program will be monitored through: (i) a set of quantitative performance criteria and indicative targets (MEFP Table 1); (ii) a set of structural performance criteria and benchmarks (MEFP Table 2); and (iii) semi-annual program reviews. The quantitative targets for end-March 2007 and end-September 2007 are

\footnotetext{
${ }^{9}$ The Paris Club agreed a debt treatment on Cologne terms with The Gambia in January 2003. Implementation of that agreement was suspended following the expiration of the country's last PRGF arrangement. A new agreement is expected to be signed after approval of the new PRGF arrangement.
} 
performance criteria; those for end-June 2007 and end-December 2007 are indicative targets. Definitions of all targeted variables and reporting requirements are contained in the Technical Memorandum of Understanding attached to the authorities letter of intent.

38. The Gambia has a good record of meeting its debt service obligations to the Fund, and is expected to continue in that vein. The debt service associated with the new disbursements from the Fund is relatively small over the medium-term; about 0.5 percent of total external debt service and 0.1 percent of exports (Table 9).

\section{Risks to the Program}

39. Persistently high real interest rates, shortfalls in donor support, and exogenous shocks pose the main risks to program objectives. Real interest rates are unlikely to continue falling if fiscal discipline breaks down, resulting in more domestic borrowing than programmed. Given The Gambia's past record of stop-go policies, maintaining fiscal discipline will require commitment to achieving the program's objectives by the highest political authorities. President Jammeh assured the mission of his government's commitment to the program. In the event interest payments remain high in spite of the authorities' best efforts, the program's fiscal objectives will be safeguarded by new measures to boost revenues or cut non-PRSP related expenditures.

40. A substantial shortfall in donor support would jeopardize attainment of the authorities' growth and poverty-reduction objectives, but is unlikely to affect the program's fiscal targets in the short-term. The program assumes that external assistance is provided predominantly in the form of project aid; hence expenditures are predicated on the availability of the associated external financing. Donor engagement will depend to some extent on the authorities' sustained commitment to economic reforms. In the past, some donors have also withheld support because of concerns about political freedoms and governance issues.

41. Shocks that would be most damaging to program objectives are those that adversely impact tourism and trade - e.g., political instability or border closures. Adverse weather conditions would also lower growth and the welfare of farmers, but would not be as damaging to achieving short-term program targets. Responses to adverse shocks will be discussed in the context of program reviews.

\section{Staff APPRAISAL}

42. Macroeconomic performance has improved significantly over the last few years. The authorities implemented strong financial policies to stabilize the economy following serious policy slippages in the early 2000s. They deserve credit for achieving low inflation, stabilizing the exchange rate, and rebuilding international reserves. 
43. The program is aligned with the objectives set out in the new PRSP. The staff welcomes steps to integrate the PRSP into the budget process, and to safeguard povertyreducing expenditure.

44. The program's policies are appropriately designed to bring down the public debt to a sustainable level and consolidate macroeconomic stability. A reduction of domestic debt is essential to bring down interest rates and ease the debt-service burden. The program also rightly makes allowance for a speedy repayment of accumulated domestic arrears. Staff welcomes the measures taken to improve revenue collection and administration.

45. After much delay, the authorities are moving aggressively with plans to improve public financial management. The launching of IFMIS in January should facilitate the establishment of a comprehensive commitment control system by mid-2007, and enhance transparency and accountability in public financial management.

46. Monetary policy is appropriately prudent at this stage. The policy stance will be re-assessed as part of the first program review, against the backdrop of fiscal developments and the evolution of interest rates.

47. The CBG has made progress toward strengthening internal controls, however vulnerabilities remain in key safeguards areas. The program includes conditionality on priority actions based on the update Safeguards Assessment. Staff encourages the CBG to implement the recommendations of the update Safeguards Assessment.

48. Financial intermediation needs deepening. The licensing of two new banks and the plan to establish a credit bureau should help, but legal reforms are needed to remove disincentives to lending.

49. Staff recommends approval of the authorities' request for a new three-year arrangement under the PRGF and for interim relief under the HIPC initiative. 
Table 1. The Gambia: Selected Economic and Financial Indicators, 2004-11

\begin{tabular}{|c|c|c|c|c|c|c|c|c|}
\hline & 2004 & 2005 & $\begin{array}{r}2006 \\
\text { Est. }\end{array}$ & $\begin{array}{l}2007 \\
\text { Proj. }\end{array}$ & $\begin{array}{l}2008 \\
\text { Proj. }\end{array}$ & $\begin{array}{l}2009 \\
\text { Proj. }\end{array}$ & $\begin{array}{l}2010 \\
\text { Proj. }\end{array}$ & $\begin{array}{l}2011 \\
\text { Proj. }\end{array}$ \\
\hline & \multicolumn{8}{|c|}{ (Annual percentage changes, unless otherwise indicated) } \\
\hline Nominal GDP (millions of dalasis) & 12,042 & 13,182 & 14,248 & 15,732 & 17,261 & 18,894 & 20,637 & 22,540 \\
\hline Nominal GDP & 20.1 & 9.5 & 8.1 & 10.4 & 9.7 & 9.5 & 9.2 & 9.2 \\
\hline GDP at constant prices & 7.0 & 5.1 & 6.5 & 7.0 & 6.0 & 6.0 & 6.0 & 6.0 \\
\hline \multicolumn{9}{|l|}{ External sector } \\
\hline Exports, f.o.b. ${ }^{1}$ & 10.5 & -5.7 & 31.5 & 3.4 & 6.3 & 1.5 & 10.0 & 4.9 \\
\hline Of which: domestic exports & 47.5 & -72.4 & 276.7 & 3.9 & 7.3 & 5.0 & 11.1 & 11.8 \\
\hline Imports, f.o.b. ${ }^{1}$ & 46.2 & 9.8 & 11.8 & 10.8 & 3.7 & 3.0 & 6.5 & 3.1 \\
\hline Terms of trade ${ }^{2}$ & -10.2 & -14.3 & -7.5 & 2.2 & 3.5 & 3.4 & 3.2 & 1.8 \\
\hline Nominal effective exchange rate (period average) ${ }^{3}$ & -11.3 & 4.2 & 1.9 & $\ldots$ & $\ldots$ & $\ldots$ & $\ldots$ & $\ldots$ \\
\hline Net foreign assets & 28.8 & 0.3 & 7.4 & 9.9 & 3.9 & 4.0 & 2.8 & 2.9 \\
\hline Net domestic assets & -10.5 & 12.8 & 9.1 & 3.3 & 8.1 & 6.1 & 7.1 & 6.9 \\
\hline Credit to the government (net) ${ }^{4}$ & -10.6 & 6.3 & 6.2 & 0.0 & -1.2 & -0.9 & -2.2 & -2.7 \\
\hline Credit to the private sector and public enterprises & -6.5 & 5.5 & 9.1 & 5.9 & 5.9 & 5.7 & 6.0 & 6.1 \\
\hline Claims on foreign exchange bureaus & -1.2 & 0.0 & -0.6 & -0.4 & -0.3 & -0.2 & -0.2 & -0.1 \\
\hline Other items net & 7.7 & 1.0 & -5.6 & -2.2 & 3.7 & 1.5 & 3.5 & 3.6 \\
\hline Velocity (GDP/average broad money) & 2.4 & 2.3 & 2.1 & 2.1 & 2.0 & 2.0 & 2.0 & 2.0 \\
\hline Yield on treasury bills (percent per year) ${ }^{5}$ & 28.0 & 12.5 & 10.7 & $\ldots$ & $\ldots$ & $\cdots$ & $\ldots$ & $\ldots$ \\
\hline Gross domestic investment and savings & \multicolumn{8}{|c|}{ (Percent of GDP) } \\
\hline Gross investment & 26.7 & 25.7 & 23.1 & 26.2 & 23.1 & 22.4 & 21.3 & 20.2 \\
\hline Gross domestic savings & 0.8 & -2.6 & 1.0 & 3.6 & 2.9 & 3.3 & 3.5 & 3.8 \\
\hline Net domestic financing & 0.5 & 3.7 & 2.4 & -2.5 & -1.5 & -0.8 & -2.0 & -2.0 \\
\hline Stock of domestic public debt & 32.9 & 35.5 & 31.6 & 30.3 & 26.4 & 23.1 & 19.0 & 15.2 \\
\hline \multicolumn{9}{|l|}{ External sector } \\
\hline \multicolumn{9}{|l|}{ Current account balance } \\
\hline Excluding official transfers & -21.2 & -25.3 & -18.3 & -21.9 & -19.3 & -17.7 & -16.8 & -15.6 \\
\hline \multirow{2}{*}{ Including official transfers } & -12.6 & -20.2 & -14.3 & -12.9 & -11.7 & -10.7 & -10.4 & -9.7 \\
\hline & \multicolumn{8}{|c|}{ (Millions of U.S. dollars, unless otherwise indicated) } \\
\hline \multicolumn{9}{|l|}{ Current account balance } \\
\hline Excluding official transfers & -84.9 & -116.7 & -92.5 & -121.9 & -117.0 & -116.4 & -119.8 & -121.0 \\
\hline Including official transfers & -50.7 & -93.0 & -72.7 & -72.1 & -70.7 & -70.5 & -74.1 & -75.4 \\
\hline Overall balance of payments & 32.9 & 14.6 & 8.9 & 16.1 & -0.6 & 1.1 & -1.6 & 0.2 \\
\hline Gross official reserves & 84.0 & 96.6 & 101.5 & 109.5 & 120.5 & 133.0 & 139.0 & 143.0 \\
\hline In months of imports, c.i.f. & 4.3 & 4.5 & 4.2 & 4.1 & 4.3 & 4.6 & 4.6 & 4.6 \\
\hline \multicolumn{9}{|l|}{ External public debt } \\
\hline Stock & 580.7 & 614.7 & 633.3 & 249.9 & 254.8 & 263.1 & 271.2 & 280.1 \\
\hline Stock (percent of GDP) & 144.8 & 133.2 & 125.0 & 44.9 & 42.1 & 40.0 & 37.9 & 36.0 \\
\hline Net present value of debt (percent of exports) ${ }^{8}$ & $\ldots$ & 215.4 & 128.0 & 82.7 & 82.2 & 84.7 & 84.3 & 85.7 \\
\hline External debt service (percent of exports) ${ }^{8}$ & 21.0 & 24.0 & 20.8 & 17.2 & 10.1 & 12.9 & 10.1 & 8.9 \\
\hline Use of Fund resources & \multicolumn{8}{|c|}{ (Millions of SDRs) } \\
\hline Purchases/disbursements & 0.0 & 0.0 & 0.0 & 4.0 & 4.0 & 4.0 & 2.0 & 0.0 \\
\hline Repurchases/repayments & -7.6 & -1.4 & -2.7 & -11.4 & 0.0 & 0.0 & 0.0 & 0.0 \\
\hline Credit outstanding & 15.9 & 14.6 & 11.8 & & $\ldots$ & & $\ldots$ & $\ldots$ \\
\hline
\end{tabular}

Sources: Gambian authorities; and IMF staff estimates and projections.

${ }^{1}$ Computed based on values in U.S. dollars.

${ }^{2}$ Excluding reexports and imports for reexport.

${ }^{3}$ Data for 2006 represent as of October 2006.

${ }^{4}$ Including advances to the government in foreign currencies.

${ }^{5}$ Weighted average for all maturities based on weekly auction data. Data shown for 2006 are for November.

${ }^{6}$ Defined as domestic revenue minus expenditure and net lending, excluding externally financed capital expenditure.

7 Defined as domestic revenue minus expenditure and net lending, excluding interest payments and externally financed capital expenditure.

${ }^{8}$ Exports of goods and nonfactor services (not including re-exports). 
Table 2. The Gambia: Central Government Operations, 2004-09 (Millions of dalasis, unless otherwise indicated)

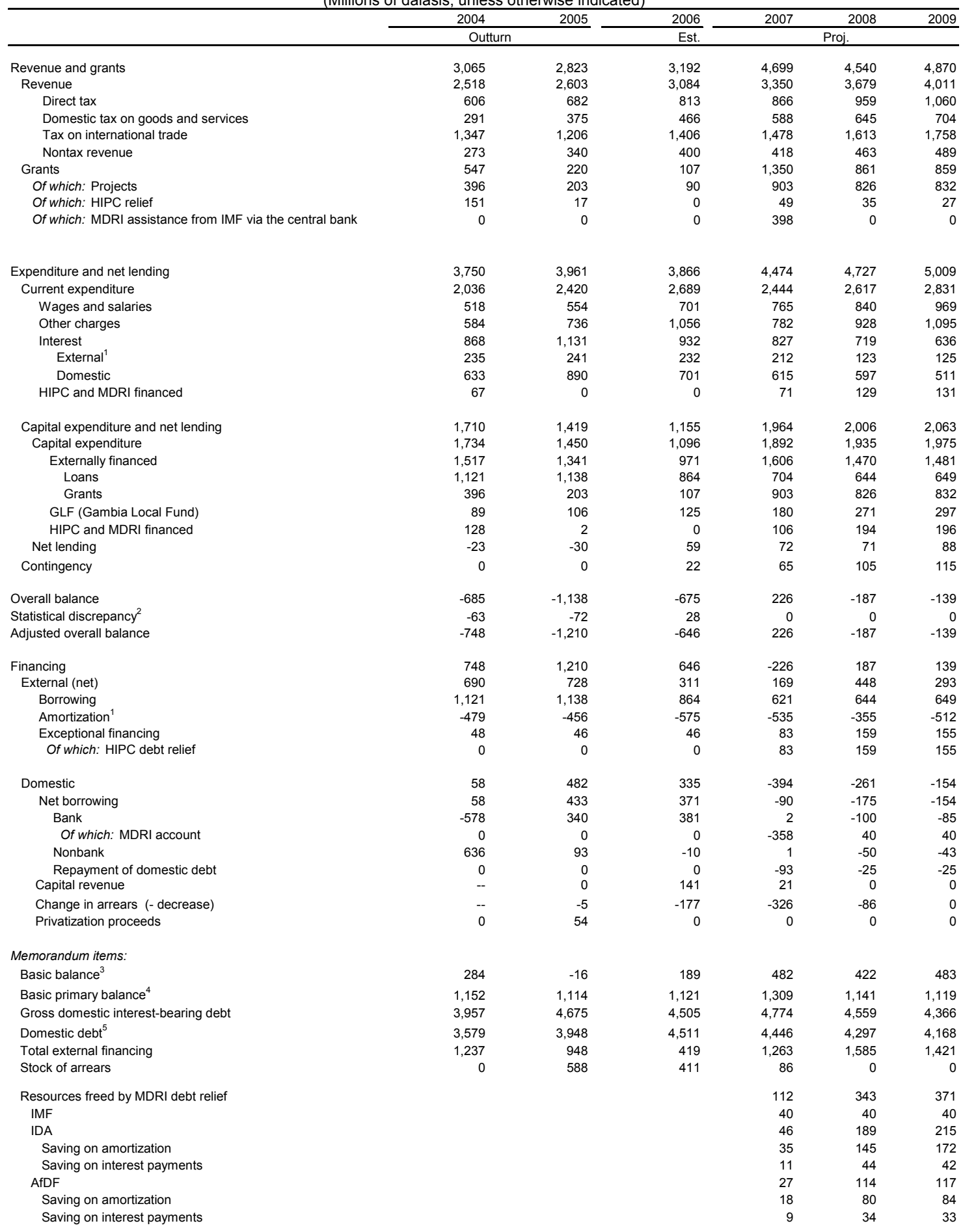

\footnotetext{
Sources: Gambian authorities; IMF staff estimates and projections.

${ }^{1}$ After MDRI debt relief from 2007 onward.

${ }^{2}$ The difference between financing and the overall balance of revenue and expenditure.

${ }^{3}$ Domestic revenue - expenditure and net lending, excluding externally financed capital spending.

${ }^{4}$ Domestic revenue - expenditure and net lending, excluding interest payments and externally financed capital spending

${ }^{5}$ Including non-interest-bearing notes held by the CBG, net of balances in the sterilization account (which holds the proceeds from treasury bill sales),

and net of balances held in the MDRI account.
} 
Table 2. The Gambia: Central Government Operations, 2004-09 (continued)

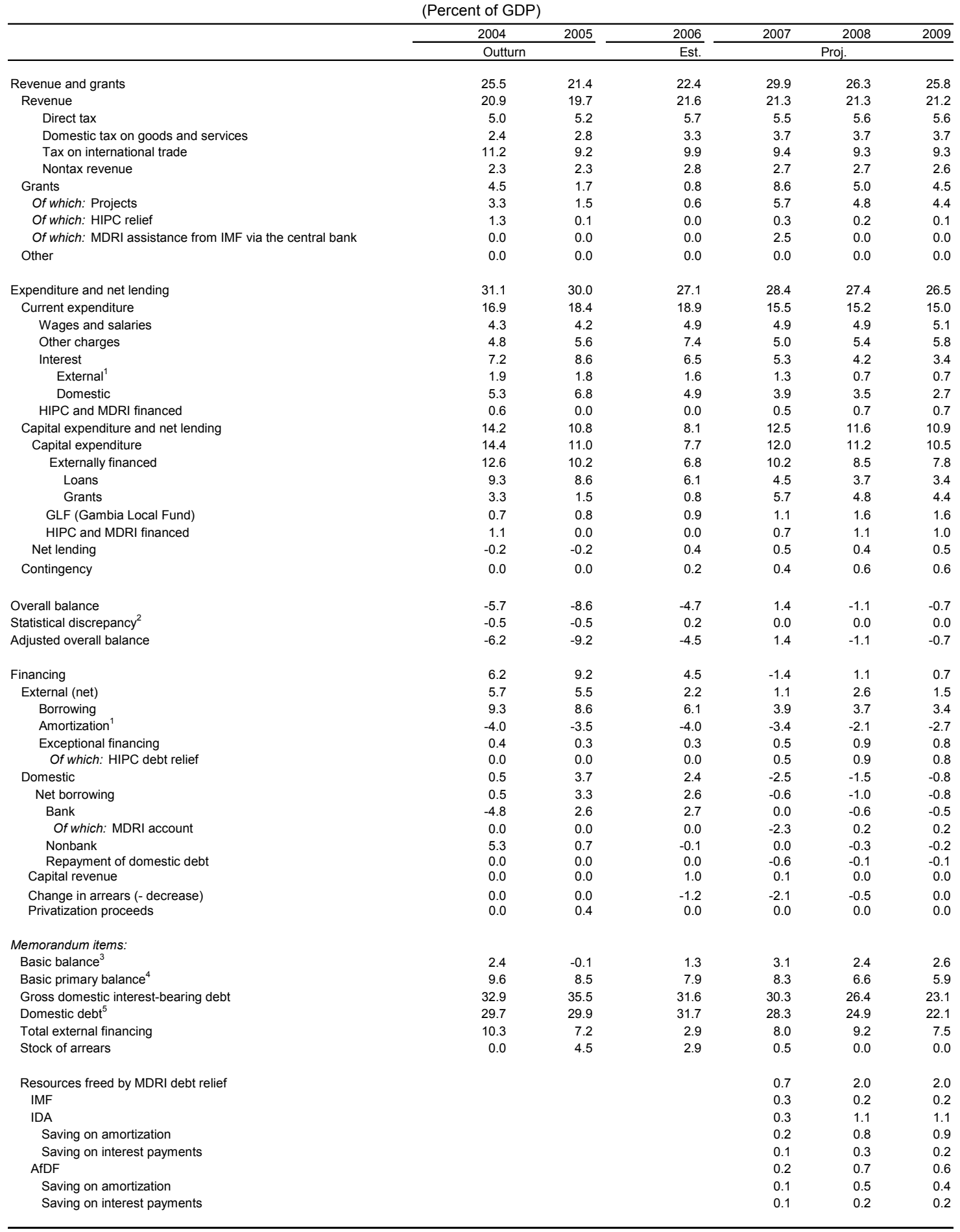

Sources: Gambian authorities; IMF staff estimates and projections.

${ }^{1}$ After MDRI debt relief from 2007 onward.

${ }^{2}$ The difference between financing and the overall balance of revenue and expenditure.

${ }^{3}$ Domestic revenue - expenditure and net lending, excluding externally financed capital spending

${ }^{4}$ Domestic revenue - expenditure and net lending, excluding interest payments and externally financed capital spending.

${ }^{5}$ Including non-interest-bearing notes held by the CBG, net of balances in the sterilization account (which holds the proceeds from treasury bill sales),

and net of balances held in the MDRI account. 
Table 3. The Gambia: Monetary Survey, 2004-09

(Millions of dalasis, unless otherwise indicated)

\begin{tabular}{|c|c|c|c|c|c|c|}
\hline & $\begin{array}{c}2004 \\
\text { Dec. }\end{array}$ & $\begin{array}{c}2005 \\
\text { Dec. }\end{array}$ & $\begin{array}{r}2006 \\
\text { Dec. } \\
\text { Est. }\end{array}$ & $\begin{array}{c}2007 \\
\text { Dec. } \\
\text { Proj. }\end{array}$ & $\begin{array}{c}2008 \\
\text { Dec. } \\
\text { Proj. }\end{array}$ & $\begin{array}{c}2009 \\
\text { Dec. } \\
\text { Proj. }\end{array}$ \\
\hline Net foreign assets & 3,184 & 3,201 & 3,654 & 4,365 & 4,680 & 5,039 \\
\hline Net domestic assets & 2,247 & 2,944 & 3,505 & 3,744 & 4,405 & 4,958 \\
\hline Domestic credit & 2,870 & 3,510 & 4,417 & 4,811 & 5,168 & 5,582 \\
\hline Claims on government (net) & 1,009 & 1,348 & 1,729 & 1,731 & 1,631 & 1,545 \\
\hline Claims on government (net) ${ }^{1}$ & 434 & 774 & 1,653 & 1,655 & 1,581 & 1,520 \\
\hline Advances to the government in foreign currencies ${ }^{2}$ & 575 & 575 & 76 & 76 & 51 & 25 \\
\hline Claims on the private sector and public enterprises ${ }^{3}$ & 1,677 & 1,979 & 2,541 & 2,962 & 3,443 & 3,962 \\
\hline Claims on public enterprises & 223 & 260 & 265 & 270 & 276 & 281 \\
\hline Claims on private sector & 1,454 & 1,719 & 2,276 & 2,692 & 3,167 & 3,681 \\
\hline Claims on foreign exchange bureaus ${ }^{4}$ & 183 & 183 & 147 & 117 & 94 & 75 \\
\hline Other items (net) & -622 & -566 & -911 & $-1,067$ & -763 & -624 \\
\hline Broad money & 5,432 & 6,145 & 7,160 & 8,109 & 9,085 & 9,997 \\
\hline Currency outside banks & 1,416 & 1,424 & 1,647 & 1,703 & 1,908 & 2,099 \\
\hline Deposits & 4,016 & 4,721 & 5,513 & 6,406 & 7,177 & 7,898 \\
\hline \multicolumn{7}{|l|}{ Memorandum items: } \\
\hline Nominal GDP (calendar year) & 12,043 & 13,182 & 14,248 & 15,732 & 17,261 & 18,894 \\
\hline (percentage change) & 20.12 & 9.46 & 8.08 & 10.42 & 9.72 & 9.46 \\
\hline Velocity (GDP/average broad money) & 2.40 & 2.28 & 2.14 & 2.06 & 2.01 & 1.98 \\
\hline Velocity (GDP/end-of-period broad money) & 2.22 & 2.15 & 1.99 & 1.94 & 1.90 & 1.89 \\
\hline Contribution to growth of broad money & \multicolumn{6}{|c|}{ (Percent change in beginning-of-year broad money, unless otherwise indicated) } \\
\hline Broad money & 18.3 & 13.1 & 16.5 & 13.3 & 12.0 & 10.0 \\
\hline Net foreign assets & 28.8 & 0.3 & 7.4 & 9.9 & 3.9 & 4.0 \\
\hline Net domestic assets & -10.5 & 12.8 & 9.1 & 3.3 & 8.1 & 6.1 \\
\hline Domestic credit & -18.3 & 11.8 & 14.8 & 5.5 & 4.4 & 4.6 \\
\hline Claims on government (net) & -10.6 & 6.3 & 6.2 & 0.0 & -1.2 & -0.9 \\
\hline Claims on government (net) ${ }^{1}$ & -12.6 & 6.3 & 14.3 & 0.0 & -0.9 & -0.7 \\
\hline Advances to the government in foreign currencies ${ }^{2}$ & 2.0 & 0.0 & -8.1 & 0.0 & -0.3 & -0.3 \\
\hline Claims on the private sector and public enterprises ${ }^{3}$ & -6.5 & 5.5 & 9.1 & 5.9 & 5.9 & 5.7 \\
\hline Claims on public enterprises & -2.6 & 0.7 & 0.1 & 0.1 & 0.1 & 0.1 \\
\hline Claims on private sector & -3.9 & 4.9 & 9.1 & 5.8 & 5.9 & 5.7 \\
\hline Claims on foreign exchange bureaus ${ }^{4}$ & -1.2 & 0.0 & -0.6 & -0.4 & -0.3 & -0.2 \\
\hline Other items (net) & 7.7 & 1.0 & -5.6 & -2.2 & 3.7 & 1.5 \\
\hline \multicolumn{7}{|l|}{ Memorandum items: } \\
\hline \multicolumn{7}{|l|}{ Credit to the private sector and public enterprises } \\
\hline Twelve-month change (percent) & -15.1 & 18.0 & 28.4 & 16.6 & 16.2 & 15.1 \\
\hline Percent of GDP & 13.9 & 15.0 & 17.8 & 18.8 & 19.9 & 21.0 \\
\hline \multicolumn{7}{|l|}{ Selected ratios (in percent) } \\
\hline Currency outside banks/broad money & 26.1 & 23.2 & 23.0 & 21.0 & 21.0 & 21.0 \\
\hline Currency outside banks/deposits & 35.3 & 30.2 & 29.9 & 26.6 & 26.6 & 26.6 \\
\hline Deposits/broad money & 73.9 & 76.8 & 77.0 & 79.0 & 79.0 & 79.0 \\
\hline Money multiplier (broad money/reserve money) & 2.64 & 2.66 & 2.83 & 2.90 & 2.95 & 2.95 \\
\hline
\end{tabular}

Sources: Gambian authorities; IMF staff estimates and projections.

${ }^{1}$ Excluding advances to the government in foreign currencies.

${ }^{2}$ These advances reflect previously unrecorded public spending and borrowing in 2001, financed by the CBG, and the previously unrecorded depletion of foreign exchange reserves in 2001-03 as reported by the authorities on October 28, 2003.

${ }^{3}$ In March 2003, the government instructed the CBG to lend the equivalent of D137 million in U.S. dollars to a newly created

public enterprise for a seismic survey of offshore oil deposits.

${ }^{4}$ Claims on foreign exchange bureaus reflect the delayed delivery of foreign currency purchased on a spot basis. 
Table 4. The Gambia: Analytical Account of the Central Bank of The Gambia (CBG), 2004-09 (Millions of dalasis, unless otherwise indicated; end of period)

\begin{tabular}{|c|c|c|c|c|c|c|}
\hline & 2004 & 2005 & $\begin{array}{r}2006 \\
\text { Est. }\end{array}$ & $\begin{array}{r}2007 \\
\text { Proj. }\end{array}$ & $\begin{array}{r}2008 \\
\text { Proj. }\end{array}$ & $\begin{array}{r}2009 \\
\text { Proj. }\end{array}$ \\
\hline Net foreign assets & $1,757.8$ & $2,132.3$ & $2,354.4$ & $2,935.3$ & $3,107.3$ & $3,309.0$ \\
\hline Foreign assets & $2,492.5$ & $2,718.2$ & $2,852.2$ & $3,105.6$ & $3,451.7$ & $3,828.8$ \\
\hline Foreign liabilities & -734.7 & -585.9 & -497.7 & -170.3 & -344.5 & -519.8 \\
\hline Net usable reserves (millions of U.S. dollars) & 55.4 & 71.1 & 77.8 & 97.6 & 102.5 & 109.0 \\
\hline Net domestic assets & 303.0 & 174.3 & 173.2 & -139.0 & -27.7 & 79.8 \\
\hline Domestic credit & 195.9 & -29.2 & -86.7 & -424.0 & -327.4 & -226.2 \\
\hline Claims on government (net) & -186.8 & -413.8 & -433.9 & -741.9 & -621.9 & -501.9 \\
\hline Claims on government (net) ${ }^{1}$ & -761.6 & -988.6 & -509.9 & -817.9 & -672.6 & -527.2 \\
\hline Advances to the government in foreign currencies ${ }^{2}$ & 574.8 & 574.8 & 76.0 & 76.0 & 50.7 & 25.3 \\
\hline Claims on banks (net) ${ }^{3}$ & 33.6 & 33.6 & 33.6 & 33.6 & 33.6 & 33.6 \\
\hline Claims on other resident sectors & 349.0 & 351.0 & 313.6 & 284.3 & 260.8 & 242.1 \\
\hline Claims on private sector & 28.8 & 30.8 & 30.1 & 30.1 & 30.1 & 30.1 \\
\hline Claims on public enterprises & 136.9 & 136.9 & 136.9 & 136.9 & 136.9 & 136.9 \\
\hline Claims on foreign exchange bureaus ${ }^{4}$ & 183.3 & 183.3 & 146.6 & 117.3 & 93.8 & 75.1 \\
\hline Other items (net) & 107.1 & 203.5 & 259.9 & 284.9 & 299.8 & 306.1 \\
\hline Of which: Holdings of government's noninterest-bearing securities & 199.0 & 199.0 & 534.9 & 534.9 & 534.9 & 534.9 \\
\hline Reserve money & $2,060.7$ & $2,306.6$ & $2,527.6$ & $2,796.3$ & $3,079.6$ & $3,388.8$ \\
\hline Currency in circulation outside banks & $1,416.3$ & $1,424.2$ & $1,646.7$ & $1,702.9$ & $1,907.8$ & $2,099.4$ \\
\hline Bank reserves & 644.5 & 882.4 & 880.9 & $1,093.3$ & $1,171.8$ & $1,289.4$ \\
\hline Cash & 69.3 & 113.4 & 176.2 & 218.7 & 234.4 & 257.9 \\
\hline Deposits at the central bank & 575.2 & 769.0 & 704.8 & 874.7 & 937.4 & $1,031.6$ \\
\hline Contribution to growth of reserve money & (Percent char & in beginnir & -year rese & noney, un & otherwise & cated) \\
\hline Reserve money & 11.0 & 11.9 & 9.6 & 10.6 & 10.1 & 10.0 \\
\hline Net foreign assets & 49.0 & 18.2 & 9.6 & 23.0 & 6.1 & 6.6 \\
\hline Net domestic assets & -38.0 & -6.2 & 0.0 & -12.4 & 4.0 & 3.5 \\
\hline Domestic credit & -55.7 & -10.9 & -2.5 & -13.3 & 3.5 & 3.3 \\
\hline Claims on government (net) & -55.4 & -11.0 & -0.9 & -12.2 & 4.3 & 3.9 \\
\hline Claims on government (net) ${ }^{1}$ & -60.3 & -11.0 & 20.8 & -12.2 & 5.2 & 4.7 \\
\hline Advances to the government in foreign currencies ${ }^{2}$ & 5.0 & 0.0 & -21.6 & 0.0 & -0.9 & -0.8 \\
\hline Claims on banks (net) ${ }^{3}$ & 2.4 & 0.0 & 0.0 & 0.0 & 0.0 & 0.0 \\
\hline Claims on private and public enterprises & 0.2 & 0.1 & 0.0 & 0.0 & 0.0 & 0.0 \\
\hline Claims on public enterprises & 0.0 & 0.0 & 0.0 & 0.0 & 0.0 & 0.0 \\
\hline Claims on private enterprises & 0.2 & 0.1 & 0.0 & 0.0 & 0.0 & 0.0 \\
\hline Claims on foreign exchange bureaus ${ }^{4}$ & -2.9 & 0.0 & -1.6 & -1.2 & -0.8 & -0.6 \\
\hline Other items (net) & 17.7 & 4.7 & 2.4 & 1.0 & 0.5 & 0.2 \\
\hline \multicolumn{7}{|l|}{ Memorandum items: } \\
\hline \multicolumn{7}{|l|}{ Twelve-month change (percent) } \\
\hline Reserve money & 11.0 & 11.9 & 9.6 & 10.6 & 10.1 & 10.0 \\
\hline Net foreign asset & 107.4 & 21.3 & 10.4 & 24.7 & 5.9 & 6.5 \\
\hline Net domestic asset & -70.0 & -42.5 & -0.6 & -180.3 & -80.1 & -388.6 \\
\hline \multicolumn{7}{|l|}{ Of which: } \\
\hline Claims on government (net) & -122.2 & 121.6 & 4.8 & 71.0 & -16.2 & -19.3 \\
\hline Claims on banks (net) & -427.7 & 0.0 & 0.0 & 0.0 & 0.0 & 0.0 \\
\hline Claims on public and private enterprises & 2.5 & 1.2 & -0.4 & 0.0 & 0.0 & 0.0 \\
\hline Claims on foreign exchange bureaus & -23.0 & 0.0 & -20.0 & -20.0 & -20.0 & -20.0 \\
\hline Other items (net) & -148.4 & 90.0 & 27.7 & 9.6 & 5.2 & 2.1 \\
\hline Outstanding new PRGF funds (millions of dalasis) & & & & -170.3 & -344.5 & -519.8 \\
\hline MDRI debt relief (millions of dalasis) & & & & -415.3 & & \\
\hline Gross international reserves (millions of U.S. dollars) & 84.0 & 96.6 & 101.5 & 109.5 & 120.5 & 133.0 \\
\hline
\end{tabular}

Sources: Gambian authorities; IMF staff estimates and projections.

${ }^{1}$ Excluding advances to the government in foreign currencies.

${ }^{2}$ These advances reflect previously unrecorded public spending and borrowing in 2001, financed by the CBG, and

previously unrecorded depletion of foreign exchange reserves in 2001-03 as reported by the authorities on October 28, 2003.

${ }^{3}$ Advances to commercial banks and commercial banks' holdings of central bank bills.

${ }^{4}$ Claims on foreign exchange bureaus reflect the delayed delivery of foreign currency purchased on a spot basis. 
Table 5. The Gambia: Balance of Payments, 2004-11

(Millions of U.S. dollars, unless otherwise indicated)

\begin{tabular}{|c|c|c|c|c|c|c|c|c|}
\hline & $\begin{array}{c}2004 \\
\text { Prel. }\end{array}$ & $\begin{array}{r}2005 \\
\text { Prel. }\end{array}$ & $\begin{array}{r}2006 \\
\text { Est. }\end{array}$ & $\begin{array}{c}2007 \\
\text { Proj. }\end{array}$ & $\begin{array}{c}2008 \\
\text { Proj. }\end{array}$ & $\begin{array}{r}2009 \\
\text { Proj. }\end{array}$ & $\begin{array}{c}2010 \\
\text { Proj. }\end{array}$ & $\begin{array}{r}2011 \\
\text { Proj. }\end{array}$ \\
\hline Trade balance & -91.1 & -117.3 & -110.5 & -132.7 & -133.7 & -140.0 & -143.6 & -145.0 \\
\hline Exports, f.o.b. & 111.6 & 105.3 & 138.4 & 143.1 & 152.2 & 154.5 & 169.9 & 178.2 \\
\hline Groundnuts/groundnut products & 16.9 & 2.0 & 16.2 & 16.8 & 17.5 & 18.2 & 19.3 & 20.5 \\
\hline Other domestic exports & 9.0 & 5.2 & 10.7 & 11.1 & 12.4 & 13.2 & 15.6 & 18.6 \\
\hline Reexports & 85.8 & 98.2 & 111.6 & 115.2 & 122.2 & 123.0 & 135.0 & 139.1 \\
\hline Imports, f.o.b. & -202.8 & -222.6 & -248.9 & -275.8 & -285.9 & -294.4 & -313.5 & -323.1 \\
\hline For domestic use & -131.8 & -141.4 & -160.8 & -184.8 & -189.4 & -197.3 & -206.9 & -213.3 \\
\hline Of which: oil products & -23.2 & -29.8 & -29.5 & -33.5 & -39.0 & -41.3 & -47.5 & -51.4 \\
\hline For reexport & -71.0 & -81.2 & -88.1 & -91.0 & -96.5 & -97.2 & -106.6 & -109.9 \\
\hline Factor services (net) & 11.1 & 6.4 & 5.9 & -5.1 & -4.5 & -0.7 & -3.1 & -4.7 \\
\hline Net income ${ }^{1}$ & -35.1 & -42.1 & -45.8 & -51.9 & -54.4 & -58.2 & -62.2 & -66.0 \\
\hline Net remittances & 46.2 & 48.5 & 51.7 & 46.8 & 50.0 & 57.5 & 59.0 & 61.3 \\
\hline Nonfactor services & -12.9 & -13.2 & -1.7 & 6.6 & 11.6 & 14.2 & 16.4 & 17.5 \\
\hline Of which: travel income (net) & 47.1 & 49.6 & 64.8 & 77.4 & 83.2 & 87.6 & 91.3 & 93.8 \\
\hline Private unrequited transfers (net) & 8.0 & 7.4 & 13.8 & 9.2 & 9.7 & 10.1 & 10.6 & 11.1 \\
\hline Official unrequited transfers (net) & 34.2 & 23.7 & 19.8 & 49.7 & 46.2 & 45.9 & 45.7 & 45.6 \\
\hline \multicolumn{9}{|l|}{ Current account balance } \\
\hline Excluding official transfers & -84.9 & -116.7 & -92.5 & -121.9 & -117.0 & -116.4 & -119.8 & -121.0 \\
\hline Including official transfers & -50.7 & -93.0 & -72.7 & -72.1 & -70.7 & -70.5 & -74.1 & -75.4 \\
\hline Capital and financial account & 79.8 & 103.6 & 81.9 & 88.3 & 70.1 & 71.6 & 72.5 & 75.6 \\
\hline IMF MDRI Relief & & & & 14.1 & & & & \\
\hline MDRI Debt forgiveness (IDA, AfDF loans) & & & & 373.0 & & & & \\
\hline Official loans (net) & 23.0 & 25.5 & 10.3 & 3.0 & 10.1 & 4.7 & 8.4 & 9.9 \\
\hline Project-related & 37.3 & 39.8 & 30.7 & 22.0 & 22.6 & 22.6 & 22.6 & 22.5 \\
\hline Program loans & 0.0 & 0.0 & 0.0 & 0.0 & 0.0 & 0.0 & 0.0 & 0.0 \\
\hline Amortization $^{1}$ & -14.4 & -14.4 & -20.5 & -19.0 & -12.5 & -17.8 & -14.2 & -12.6 \\
\hline Private capital inflow & 56.8 & 78.1 & 71.6 & 71.1 & 60.0 & 66.9 & 64.1 & 65.6 \\
\hline Foreign direct investment (net) & 47.6 & 60.8 & 73.9 & 69.1 & 60.6 & 66.2 & 68.2 & 68.9 \\
\hline Other investment (net) & 9.2 & 17.3 & -2.2 & 2.1 & -0.6 & 0.8 & -4.0 & -3.3 \\
\hline Of which: supplier's credits & 6.3 & 8.3 & 5.6 & 6.2 & 6.4 & 6.6 & 7.0 & 7.3 \\
\hline Other official investment liabilities (IDA, AfDF loans) & & & & -373.0 & & & & \\
\hline Errors and omissions & 2.2 & 2.4 & -1.9 & 0.0 & 0.0 & 0.0 & 0.0 & 0.0 \\
\hline Overall balance & 31.3 & 13.0 & 7.3 & 16.1 & -0.6 & 1.1 & -1.6 & 0.2 \\
\hline Financing & -31.3 & -13.0 & -7.3 & -16.1 & 0.6 & -1.1 & 1.6 & -0.2 \\
\hline Change in gross official reserves (increase $=-$ ) & -21.7 & -12.6 & -4.9 & -8.0 & -11.0 & -12.5 & -6.0 & -4.0 \\
\hline \multicolumn{9}{|l|}{ Use of IMF resources } \\
\hline Repayments & -11.2 & -2.0 & -4.0 & -17.1 & 0.0 & 0.0 & 0.0 & 0.0 \\
\hline Disbursements & 0.0 & 0.0 & 0.0 & 6.0 & 6.0 & 6.0 & 3.0 & 0.0 \\
\hline Exceptional financing ${ }^{2}$ & 1.6 & 1.6 & 1.6 & 2.9 & 5.6 & 5.4 & 4.6 & 3.8 \\
\hline \multicolumn{9}{|l|}{ Memorandum items: } \\
\hline \multicolumn{9}{|l|}{ Current account balance (percent of GDP) } \\
\hline Excluding official transfers & -21.2 & -25.3 & -18.3 & -21.9 & -19.3 & -17.7 & -16.8 & -15.6 \\
\hline Including official transfers & -12.6 & -20.2 & -14.3 & -12.9 & -11.7 & -10.7 & -10.4 & -9.7 \\
\hline \multicolumn{9}{|l|}{ Gross official reserves (end of period) } \\
\hline In millions of U.S. dollars & 84.0 & 96.6 & 101.5 & 109.5 & 120.5 & 133.0 & 139.0 & 143.0 \\
\hline In months of imports, c.i.f. & 4.3 & 4.5 & 4.2 & 4.1 & 4.3 & 4.6 & 4.6 & 4.6 \\
\hline
\end{tabular}

Sources: Gambian authorities; IMF staff estimates and projections.

${ }^{1}$ Reflects the impact of MDRI stock relief from IDA and AfDF from 2007.

${ }^{2}$ Includes arrears and debt relief. From 2007, includes debt relief from Paris Club; interim relief from multilaterals is treated as grants. 
Table 6. The Gambia: Financial Soundness Indicators for the Banking Sector, 2000September 2006

(Percent at end-of-period, unless otherwise indicated)

\begin{tabular}{|c|c|c|c|c|c|c|c|}
\hline & 2000 & 2001 & 2002 & 2003 & 2004 & 2005 & $\begin{array}{l}\text { Sep. } \\
2006\end{array}$ \\
\hline \multicolumn{8}{|l|}{ Capital adequacy } \\
\hline Regulatory capital to risk-weighted assets & 22 & 21 & 18 & 17 & 20 & 54 & 46 \\
\hline Percentage of banks greater or equal to 10 percent & 80 & 100 & 83 & 100 & 67 & 100 & 100 \\
\hline Percentage of banks below 10 and above 6 percent minimum & 20 & 0 & 17 & 0 & 33 & 0 & 0 \\
\hline Percentage of banks below 6 percent minimum & 0 & 0 & 0 & 0 & 0 & 0 & 0 \\
\hline Capital (net worth) to assets & 16 & 11 & 12 & 13 & 12 & 12 & 13 \\
\hline \multicolumn{8}{|l|}{ Asset quality } \\
\hline Foreign exchange loans to total loans & $\cdots$ & $\ldots$ & $\ldots$ & $\ldots$ & $\ldots$ & $\ldots$ & $\ldots$ \\
\hline Past-due loans to gross loans & 12 & 10 & $\dddot{7}$ & 15 & $\dddot{11}$ & $\dddot{12}$ & 13 \\
\hline Nonperforming loans (in millions of dalasi) & 85 & 85 & 89 & 271 & 182 & 238 & 302 \\
\hline Watch-listed loans & $\ldots$ & $\ldots$ & $\ldots$ & $\ldots$ & $\ldots$ & $\ldots$ & $\ldots$ \\
\hline Provision (percent of past-due loans) & 85 & 86 & 82 & 42 & 80 & 74 & 66 \\
\hline \multicolumn{8}{|l|}{ Earnings and profitability } \\
\hline Net profit (before tax)/net income & 152 & 148 & 148 & 126 & 154 & 153 & 152 \\
\hline Return on assets ${ }^{1}$ & 10 & 6 & 11 & 20 & 9 & 6 & 4 \\
\hline Return on equity ${ }^{2}$ & 80 & 46 & 72 & 142 & 76 & 48 & 35 \\
\hline Expense/income & $\ldots$ & $\ldots$ & $\ldots$ & $\ldots$ & $\ldots$ & $\ldots$ & $\ldots$ \\
\hline \multicolumn{8}{|l|}{ Interest rate spread (deposit money banks) } \\
\hline Lending rate minus demand deposit rates ${ }^{3}$ & 14.0 & 14.0 & 13.5 & 22.3 & 22.3 & 23.4 & 21.37 \\
\hline Saving deposit rates & $8-10$ & $8-10$ & $8-10$ & $8-17$ & $10-17$ & $5-10$ & $5-8$ \\
\hline \multicolumn{8}{|l|}{ Liquidity } \\
\hline Liquid assets/total assets & $\ldots$ & $\ldots$ & 28 & 36 & 40 & 57 & $\ldots$ \\
\hline Liquid assets/short-term liabilities & $\ldots$ & $\ldots$ & $\ldots$ & $\ldots$ & $\ldots$ & $\ldots$ & $\ldots$ \\
\hline Loan/deposits & 46 & 37 & 50 & 43 & 34 & 42 & 43 \\
\hline Liquid assets/total deposits & $\ldots$ & $\ldots$ & 41 & 47 & 53 & 79 & 61 \\
\hline Foreign exchange liabilities/total liabilities & $\ldots$ & $\ldots$ & $\ldots$ & $\ldots$ & $\ldots$ & $\ldots$ & $\ldots$ \\
\hline Excess reserves/broad money ${ }^{4}$ & 2.13 & 1.78 & 3.17 & 0.52 & 2.24 & 3.27 & 1.09 \\
\hline \multicolumn{8}{|l|}{ Sensitivity to market risk } \\
\hline Net foreign exchange assets (liabilities) to shareholders' funds & $\ldots$ & $\ldots$ & 75 & 43 & 85 & 94 & $\ldots$ \\
\hline
\end{tabular}

Sources: Gambian authorities; IMF staff estimates.

${ }^{1}$ Net income before extraordinary items and taxes divided by the average value of total assets (average of current and preceding end-year values).

${ }^{2}$ Net income before extraordinary items and taxes divided by the average value of total capital and reserves (average of current and preceding end-year values).

${ }^{3}$ Based on mid-rate for the range of lending rates.

${ }^{4}$ Based on excess reserves for the end of the last week of each month as provided by the banks, and broad money as of month's end. 
Table 7. The Gambia: HIPC Initiative Completion Point Triggers

\begin{tabular}{|c|c|}
\hline Measures & Status \\
\hline $\begin{array}{l}\text { 1. Poverty reduction } \\
\text { (i) A full PRSP has been prepared through a participatory process and satisfactorily } \\
\text { implemented for one year, as evidenced by the Joint Staff Assessment of the country's } \\
\text { annual progress report. }\end{array}$ & Met \\
\hline $\begin{array}{l}\text { (ii) Improvement of the poverty data base and monitoring capacity, as evidenced by } \\
\text { progress in restructuring the Central Statistics Department or developing its capacity. }\end{array}$ & In progress \\
\hline $\begin{array}{l}\text { 2. Macroeconomic stability } \\
\text { Continued maintenance of macroeconomic stability as evidenced by satisfactory } \\
\text { implementation of the PRGF-supported program. }\end{array}$ & $\begin{array}{l}\text { Not met. Requires completion of one } \\
\text { review under a new PRGF and at } \\
\text { least six months of implementation. }\end{array}$ \\
\hline $\begin{array}{l}\text { 3. Governance } \\
\text { Progress in strengthening public expenditure management as evidenced by (i)the issuance } \\
\text { of annual public reports on budget execution; and (ii) semiannual reports on the use of } \\
\text { interim HIPC Initiative debt relief, the latter to be reviewed by the Task Force and the High- } \\
\text { Level Economic Committee (HILEC). }\end{array}$ & $\begin{array}{l}\text { (i) In progress (draft report for } 2005 \\
\text { has been prepared). } \\
\text { (ii) In progress }\end{array}$ \\
\hline $\begin{array}{l}\text { 4. Social sector reforms } \\
\text { (i) Budgetary savings from HIPC interim debt service will be used in accordance with the } \\
\text { annual budgets approved by the Task Force and the HILEC. }\end{array}$ & Not met \\
\hline $\begin{array}{l}\text { (ii) Measures and targets regarding progress in implementing education and health reform } \\
\text { programs include: }\end{array}$ & \\
\hline $\begin{array}{l}\text { (a) Increase by at least } 45 \text { percent (from } 192 \text { graduates in the base academic } \\
\text { year 2000/01) the number of teachers for lower basic education graduating from The } \\
\text { Gambia College; this measure will help The Gambia raise the quality of teaching in the most } \\
\text { important grades. }\end{array}$ & Met \\
\hline $\begin{array}{l}\text { (b) Ensure appropriate funding of a trust fund for girls' scholarships in the poorest } \\
\text { regions and make progress in raising such rates by expanding this scholarship scheme to } \\
\text { no less than } 2,000 \text { girls annually in at least } 3 \text { regions. }\end{array}$ & Met \\
\hline $\begin{array}{l}\text { (c) Increase by at least } 5 \text { percent each year (from } 44 \text { percent in the base year } \\
\text { of 1998) the number of births attended by a person trained in antenatal care. This should } \\
\text { reduce the relatively high maternal death rate; monitoring mechanisms for this indicator } \\
\text { have also been defined in the Participatory Health, Population, and Nutrition Project } \\
\text { (PHPNP). }\end{array}$ & Met \\
\hline $\begin{array}{l}\text { (d) Increase the share of primary and secondary health care within the recurrent } \\
\text { budget for health. The recurrent budget for primary and secondary health care is } \\
\text { understood to comprise health centers; dispensaries and subdispensaries; health promotion } \\
\text { and protection; family health; disease control; and nurses' training. These are covered by } \\
\text { budget lines } 06 \text { to } 11 \text { under heading } 21 \text { of the budget; they do not include expenditures } \\
\text { incurred directly or indirectly on (i) foreign personnel; and (ii) all the referral hospitals. The } \\
\text { base year for measurement is } 1999 \text {. }\end{array}$ & In progress \\
\hline \multicolumn{2}{|l|}{$\begin{array}{l}\text { 5. Structural reforms } \\
\text { Measures to promote private sector development: }\end{array}$} \\
\hline (i) Establish a functional multisector regulatory agency. & Met \\
\hline (ii) Bring to the point of sale The Gambia's two major public groundnut processing plants. & In progress \\
\hline
\end{tabular}


Table 8. The Gambia: Proposed Schedule of Disbursements

\begin{tabular}{|c|c|c|c|}
\hline \multirow[b]{2}{*}{ Period } & \multicolumn{2}{|c|}{ Disbursement } & \multirow[b]{2}{*}{ Conditions } \\
\hline & SDRs & $\begin{array}{l}\text { Percent } \\
\text { of quota }^{1}\end{array}$ & \\
\hline February 21, 2007 & $2,000,000$ & 6.43 & Approval of the arrangement \\
\hline July 31, 2007 & $2,000,000$ & 6.43 & Completion of first review (end-March 2007 test date) \\
\hline January 31, 2008 & $2,000,000$ & 6.43 & Completion of second review (end-September 2007 test date) \\
\hline July 31, 2008 & $2,000,000$ & 6.43 & Completion of third review (end-March 2008 test date) \\
\hline January 31, 2009 & $2,000,000$ & 6.43 & Completion of fourth review (end-September 2008 test date) \\
\hline July 31, 2009 & $2,000,000$ & 6.43 & Completion of fifth review (end-March 2009 test date) \\
\hline January 30, 2010 & $2,000,000$ & 6.43 & Completion of sixth review (end-September 2009 test date) \\
\hline Total & $14,000,000$ & 45.02 & \\
\hline
\end{tabular}

${ }^{1}$ The Gambia's quota is SDR 31.10 million. 
Table 9. The Gambia: Indicators of Capacity to Repay the Fund, 2006-11 ${ }^{1}$ (Millions of U.S. dollars, unless otherwise indicated)

\begin{tabular}{|c|c|c|c|c|c|c|}
\hline & $\begin{array}{c}2006 \\
\text { Est. }\end{array}$ & $\begin{array}{l}2007 \\
\text { Proj. }\end{array}$ & $\begin{array}{l}2008 \\
\text { Proj. }\end{array}$ & $\begin{array}{l}2009 \\
\text { Proj. }\end{array}$ & $\begin{array}{l}2010 \\
\text { Proj. }\end{array}$ & $\begin{array}{l}2011 \\
\text { Proj. }\end{array}$ \\
\hline \multicolumn{7}{|l|}{ Fund credit outstanding } \\
\hline Millions of SDRs & 11.8 & 4.0 & 8.0 & 12.0 & 14.0 & 14.0 \\
\hline Millions of U.S. dollars & 17.4 & 6.0 & 12.0 & 18.0 & 21.0 & 21.1 \\
\hline Percent of quota & 37.9 & 12.9 & 25.7 & 38.6 & 45.0 & 45.0 \\
\hline Percent of exports of goods and services ${ }^{2}$ & 12.4 & 3.9 & 7.2 & 10.5 & 11.4 & 11.0 \\
\hline Percent of total external debt & 2.7 & 1.0 & 2.0 & 2.9 & 3.4 & 3.4 \\
\hline Percent of gross official reserves & 17.1 & 5.5 & 10.0 & 13.6 & 15.1 & 14.8 \\
\hline Fund obligations $1 /$ & 4.3 & 3.4 & 0.0 & 0.1 & 0.1 & 0.1 \\
\hline Total charges and interest & 0.3 & 0.4 & 0.0 & 0.1 & 0.1 & 0.1 \\
\hline Existing drawings & 0.3 & 0.3 & 0.0 & 0.0 & 0.0 & 0.0 \\
\hline Prospective drawings & 0.0 & 0.0 & 0.0 & 0.1 & 0.1 & 0.1 \\
\hline Total repayments/repurchases & 4.0 & 3.0 & 0.0 & 0.0 & 0.0 & 0.0 \\
\hline Existing drawings & 4.0 & 3.0 & 0.0 & 0.0 & 0.0 & 0.0 \\
\hline Prospective drawings & 0.0 & 0.0 & 0.0 & 0.0 & 0.0 & 0.0 \\
\hline Percent of exports of goods and services ${ }^{2}$ & 3.1 & 2.2 & 0.0 & 0.0 & 0.1 & 0.1 \\
\hline Percent of external debt service & 14.9 & 12.6 & 0.3 & 0.3 & 0.6 & 0.6 \\
\hline Percent of gross official reserves & 4.3 & 3.1 & 0.0 & 0.1 & 0.1 & 0.1 \\
\hline External debt service, percent of exports ${ }^{2}$ & 20.8 & 17.2 & 10.1 & 12.9 & 10.1 & 8.9 \\
\hline \multicolumn{7}{|l|}{ Memorandum items: } \\
\hline Exports (millions of U.S. dollars) ${ }^{2}$ & 140.1 & 155.4 & 166.1 & 172.4 & 184.4 & 192.7 \\
\hline External debt service (millions of U.S. dollars) & 29.1 & 26.7 & 16.8 & 22.2 & 18.7 & 17.1 \\
\hline Quota (millions of SDRs) & 31.1 & 31.1 & 31.1 & 31.1 & 31.1 & 31.1 \\
\hline Quota (millions of U.S. dollars) & 45.7 & 46.6 & 46.7 & 46.8 & 46.8 & 47.0 \\
\hline Gross official reserves (millions of U.S. dollars) & 101.5 & 109.5 & 120.5 & 133.0 & 139.0 & 143.0 \\
\hline Exchange rate (U.S. dollars/SDR; period average) & 1.5 & 1.5 & 1.5 & 1.5 & 1.5 & 1.5 \\
\hline
\end{tabular}

Sources: Data provided by the authorities; IMF staff estimates and projections.

${ }^{1}$ Includes the prospective disbursement under the PRGF of SDR 14 millions (45 percent of quota); data for 2007 exclude MDRI stock relief. Data beyond 2007 include MDRI relief.

${ }^{2}$ Exports of goods and nonfactor services (not including reexports). 


\section{APPENDIX I}

Banjul, The Gambia

February 7, 2007

Mr. Rodrigo de Rato

Managing Director

International Monetary Fund

Washington, D.C. 20431

Dear Mr. de Rato:

1. The macroeconomic environment in The Gambia has improved significantly over the last three years as a result of policies implemented by the government and the Central Bank of The Gambia (CBG) to reverse earlier policy lapses. Inflation has returned to low singledigit levels, the exchange rate of the dalasi has stabilized, and international reserves have been rebuilt to a comfortable level. Nevertheless, the country still faces many development challenges. A majority of the population lives below the poverty line, and the narrow production base of the economy makes it vulnerable to exogenous shocks such as drought. Furthermore, a heavy debt service burden has limited the government's scope for undertaking growth-promoting and poverty-reducing expenditures.

2. We seek the assistance of the International Monetary Fund to help us address the economic development challenges we face. In this regard, the government of The Gambia has prepared a medium-term economic and financial program to build on the recent achievement of macroeconomic stability by undertaking reforms aimed at promoting sustained growth and poverty reduction. The program draws on the government's recently completed second Poverty Reduction Strategy Paper (PRSP II).

3. As you know, The Gambia reached the decision point under the Heavily Indebted Poor Countries (HIPC) Initiative in December 2000, but has not yet been able to advance to the completion point, partly because the country's last IMF-supported program — under a Poverty Reduction and Growth Facility (PRGF) arrangement-went off-track soon after it was approved in 2002. Following the successful implementation of a staff monitored program (SMP) that spanned October 2005-March 2006, the government of The Gambia hereby requests a three-year arrangement under the Poverty Reduction and Growth Facility in an amount equivalent to SDR 14 million (45 percent of quota), to be disbursed in seven equal installments of SDR 2 million each. The first disbursement would occur upon Executive Board approval of this arrangement, and subsequent disbursements would be linked to six program reviews that are scheduled to be conducted at six-monthly intervals beginning in July 2007. In addition, we request the resumption of interim relief under the enhanced HIPC Initiative in the amount of SDR 0.36 million (20 percent of the total HIPC 
commitment) to help us cover part of the principal repayments to the IMF falling due during 2007.

4. The Memorandum of Economic and Financial Policies (MEFP) attached to this letter (Attachment I) describes the economic and financial policies as well as structural measures that the government intends to implement to meet the objectives of the program. Table 1 in the MEFP contains quantitative financial performance criteria and indicative targets, while Table 2 contains structural performance criteria and benchmarks, to be used to monitor the program. To further assist the IMF monitor the program, the government and the CBG undertake to provide in a timely manner the information specified in the Technical Memorandum of Understanding (TMU) attached to this letter (Attachment II).

5. The government believes that the policies and measures set forth in the MEFP are adequate to achieve the objectives of the program. However, it is prepared to take any further measures that may become appropriate for this purpose. During the implementation of the program, the government will consult with the Managing Director on the adoption of any measures that may be appropriate, on its own initiative, or whenever the Managing Director requests such a consultation.

6. We wish to assure you that the government of The Gambia is determined to fully implement the PRGF-supported program. In particular, we will take all necessary measures to ensure that the first program review is completed by end-July 2007 . That review will be based on the quantitative performance criteria at end-March 2007 and structural performance criteria through end-June 2007. We expect that by end-July 2007, the government would also have implemented all the measures required for The Gambia to reach completion point under the HIPC Initiative.

7. The government intends to make the contents of this letter and those of the attached MEFP and TMU available to the public, and so authorizes the IMF to arrange for these documents to be posted on the IMF website following Executive Board approval of The Gambia's request for the new PRGF arrangement.

Sincerely yours,

$/ \mathrm{s} /$

Mousa Bala Gaye

Secretary of State

Department of State for Finance and Economic Affairs /s/

Famara Jatta

Governor

Central Bank of The Gambia

Attachments: Memorandum of Economic and Financial Policies

Technical Memorandum of Understanding 


\section{ATTACHMENT I}

\section{THE GAMBIA}

\section{Memorandum of Economic and Financial Policies}

\section{INTRODUCTION AND BACKGROUND}

1. This memorandum outlines the government of The Gambia's economic and financial program under a three-year Poverty Reduction and Growth Facility (PRGF) arrangement with the International Monetary Fund (IMF). Following the successful implementation of a Staff Monitored Program (SMP) over the period October 2005-March 2006, the new program aims at consolidating macroeconomic stability and fostering the conditions for sustaining high growth and reducing poverty.

2. The focus of the SMP was on strengthening internal controls at the Central Bank of The Gambia (CBG) to underpin its operational independence, and enhancing public financial management and accountability. All the structural benchmarks under the SMP were implemented, albeit a few with a delay. In particular, the $\mathrm{CBG}$ instituted guidelines and procedures to strengthen accounting practices, and the government made progress toward clearing the backlog of unaudited accounts by submitting the 2000 and 2001 accounts to the Auditor-General. ${ }^{10}$ Performance against the quantitative targets was mixed in relation to the December 2005 targets but improved in the second half of the program and all the targets were met on a cumulative basis at the end of March 2006.

3. The Gambia reached the decision point under the enhanced Heavily Indebted Poor Countries (HIPC) Initiative in December 2000. Reaching the completion point will make The Gambia eligible for debt relief under the Multilateral Debt Relief Initiative (MDRI). The conditions for reaching the completion point include satisfactory performance under the PRGF-supported program for at least six months and the implementation of the other completion point triggers established at the decision point.

\section{Strategy for Poverty Alleviation}

4. The Gambia's first Poverty Reduction Strategy Paper (PRSP) which spanned 200205 was prepared through a broad-based participatory process. Reviews contained in two annual progress reports (APRs) prepared by the government indicated that implementation of the PRSP was hampered by capacity constraints, limited donor support, and inadequate prioritization by the government. The APRs noted some progress in increasing access to basic social services, but not much progress in the government's divestiture and decentralization programs. The APRs also highlighted a need to increase budgetary resources dedicated to PRSP-priority expenditures.

\footnotetext{
${ }^{10}$ The government subsequently submitted the 2002 and 2003 accounts to the Auditor-General in October 2006 and December 2006, respectively.
} 
5. A new PRSP (PRSP II) which integrates the achievement of the Millennium Development Goals (MDGs) into its objectives has been completed after an extensive consultative process. The strategy for growth and poverty reduction is built on the following five pillars: (i) improving the policy environment; (ii) enhancing the capacity and output of the productive sectors - agriculture, fisheries, industry, trade, and tourism; (iii) improving the delivery of basic social services; (iv) building the capacity of local communities and civil society organizations; and (v) mainstreaming issues related to gender, youth, population, HIV/AIDS, nutrition and the environment into the development process.

6. PRSP II emphasizes prudent fiscal and monetary policies to achieve macroeconomic stability. Objectives of fiscal policy include: improving revenue collection by strengthening institutional capacity, making the budget process more transparent and reflective of government priorities, and reducing the domestic debt burden by maintaining a primary surplus and containing the government's borrowing requirement. The principal objective set for monetary policy is the maintenance of low inflation. The CBG is also charged with promoting a sound and flexible financial system.

\section{RECENT ECONOMIC DEVELOPMENTS}

7. The macroeconomic environment has improved significantly over the last few years. Real GDP growth rebounded from a drought-induced decline in 2002 to an annual average of about 6 percent a year during 2003-06, outpacing average annual population growth (estimated at 2.8 percent). From a peak of 21 percent in August 2003, year-on-year consumer inflation fell to 1.8 percent in December 2005, and stayed below 2 percent through November 2006. Key interest rates have fallen, but remain very high in real terms. The CBG lowered its rediscount rate gradually from 31 percent at the end of 2004 to 15 percent in May 2006 and to 14 in October 2006. The CBG also lowered the statutory reserve requirement in October 2006-from 18 percent to 16 percent. The dalasi has remained relatively stable in nominal and real effective rate terms after marked depreciations during 2001-03.

8. Fiscal performance deteriorated in 2005 after a strong adjustment in 2004. The basic balance fell from a surplus of 2.4 percent of GDP in 2004 to near balance in 2005, reflecting extrabudgetary spending, lower customs revenues, and higher domestic interest payments. A two-month closure of the border with Senegal pushed customs revenues down, and high domestic debt at the end of 2004 resulted in a substantial increase in domestic interest payments. A larger fiscal deficit and a relatively tight monetary stance increased domestic debt from 33 percent of GDP at the end of 2004 to 36 percent of GDP at the end of 2005. Preliminary indicators of fiscal performance in 2006 show an improvement over 2005, but not by as much as envisaged in the budget mainly due to higher-than-budgeted expenditures on the hosting of the African Union summit in July 2006.

9. High interest payments have crowded out priority PRSP-related expenditures. In 2005 and 2006, interest payments are estimated to have accounted for 47 percent and 35 percent of total current expenditure, respectively. PRSP-related spending fell short of budget estimates in both 2005 and 2006. 
10. In spite of strong growth in tourism earnings, the external current account deficit (including official transfers) widened significantly from about 13 percent of GDP in 2004 to 20 percent of GDP in 2005 before falling to14 percent of GDP in 2006. The current account deficits were financed mainly by inflows of foreign direct investment (FDI) and official concessional loans. The ratio of nominal external debt to GDP fell from 145 percent at the end of 2004 to 136 percent at the end of 2005.

\section{Program ObJectives and Policies}

\section{A. Medium-Term Objectives and Macroeconomic Framework}

11. The key assumptions and objectives underlying the program's medium-term macroeconomic framework are:

- $\quad$ Annual real GDP growth of 6-7 percent.

- Annual inflation in the range of 2-4 percent.

- $\quad$ The fiscal basic balance improves from a surplus of less than 2 percent of GDP in 2006 to an annual average of about 3 percent of GDP during 2007-09.

- $\quad$ The ratio of domestic public debt to GDP is projected to fall from 32 percent at the end of 2006 to about 23 percent in 2009.

- $\quad$ The external current account deficit (including official transfers) is reduced from about 14 percent of GDP in 2006 to 9 percent of GDP in 2009.

- International reserves will be maintained at the equivalent of about four months of imports.

12. The growth rates assumed under the program are higher than the 4.5 percent average annual growth rate assumed in PRSP II. The rates for the program are based on substantial upward revisions of recent growth performance and growth prospects undertaken after completion of PRSP II.

13. The projected improvement in the fiscal basic balance reflects lower interest payments, associated with lower domestic borrowing by the government.

14. The projected reduction in the external current account deficit reflects increased official transfers and lower trade deficits. Part of the increase in official transfers is associated with a switch in the composition of official external financing for projects from loans to grants by the World Bank and the African Development Bank. In addition, it is assumed that there will be a modest increase in external financing in support of PRSP II. A donor conference has been scheduled for March 2007 to solicit additional external assistance for the implementation of PRSP II. Lower trade deficits mainly reflect a recovery in groundnut exports from near collapse in 2005. 
15. Monetary policy will target an expansion in broad money slightly above the growth rate of nominal GDP over the medium term. Low inflation is expected to boost the demand for money and steady fiscal consolidation should allow real interest rates to fall, stimulating credit to the private sector.

\section{B. Policies for $\mathbf{2 0 0 7}$}

\section{Fiscal policy and related structural reforms}

16. Fiscal policy will be the principal instrument for consolidating macroeconomic stability. The government is committed to maintaining fiscal discipline in order to contain domestic borrowing and so put downward pressure on interest rates and reduce the domestic public debt to sustainable levels.

17. The government is implementing measures to improve tax administration and thereby boost revenues through efficiency gains. The establishment of the Gambia Revenue Authority (GRA) which merged the previous Central Revenue and Customs and Excise departments under one management structure was designed to achieve synergies in the government's revenue collection efforts. Measures aimed at broadening the tax base include the introduction of taxpayer identification numbers, enhanced taxpayer education efforts, and increased staffing, training and equipment to strengthen the valuation and audit functions.

18. Weaknesses in budget formulation - for example, inadequate provision in the budget for the government's contribution to projects financed by international development banks and donors and for the payment of utility bills - have in the past led to large domestic payments arrears. Extrabudgetary expenditures have also crowded out budgeted expenditures and complicated budget execution. The government is taking steps to avoid overcommitments and the resort to extrabudgetary expenditures except in the circumstance and under the conditions stipulated in the Government Budget Management and Accountability Act (2004).

19. The launching of the Integrated Financial Management Information System (IFMIS) in January 2007 will go a long way to improve all aspects of the budget process (formulation, execution, monitoring, and reporting). In particular, commitment control will be strengthened by ensuring that funds are available before local purchase orders or other commitments are entered into. In the event IFMIS is delayed, the government will strengthen the monitoring of compliance with the manual commitment control system.

20. The government is committed to increasing poverty-reducing expenditures in line with PRSP II objectives and targets. In order to monitor the extent to which this objective is being achieved, the Department of State for Finance and Economic Affairs (DoSFEA) will continue producing a monthly report on PRSP-related expenditures out of the Gambia Local Fund, by functional and economic classifications. In due course, as IFMIS becomes established, the reporting will be extended to cover all expenditures (i.e., including those that are externally financed).

21. In order to improve the transparency and comprehensiveness of government accounts, the government is setting up a central project management and aid coordination directorate at 
DoSFEA with responsibilities that will include compiling comprehensive information on aid flows into the country and the uses to which the resources are put.

22. The government intends to enhance accountability in the use of public resources by clearing the backlog of unaudited government accounts and publishing more information on the budget and its execution. Accounts for 2004 are scheduled to be submitted to the AuditorGeneral by February 2007, and those for 2005 and 2006 will follow before the end of the year. The National Audit Office will be adequately equipped to conduct the audits and submit reports on them to the national assembly as soon as possible after they receive the accounts. With respect to publications, DoSFEA has established a website (www.dosfea.gm) where budget related information and reports will be posted.

23. In order to improve fiscal transparency and to track fiscal risks, the government will establish a reporting framework to collect quarterly financial information on selected public enterprises (see sample format in Annex I). Initially, the following public enterprises will be covered: Social Security and Housing Finance Corporation, Gambia Port Authority, and Gambia Telecommunications Cellular Company (GAMCEL). In due course, information will also be collected on the Gambia Telecommunications Company (GAMTEL) and the National Water and Electricity Company (NAWEC). This reporting framework will help to (i) maintain a transparent record of possible quasi-fiscal activities of key public enterprises, and (ii) improve the capacity of the government to monitor the financial position of parastatals and introduce, if necessary, corrective measures to improve their efficiency and reduce the risk to the budget.

\section{Monetary policy, CBG governance, and related structural reforms}

24. The CBG will continue to use a money targeting framework to pursue its price stability objective. It will also use its rediscount rate to signal changes in policy stance. In setting the rediscount rate, the CBG's Monetary Policy Committee will pay due regard to the evolution of the market determined treasury bill rates. In order to improve the efficiency of the treasury bill market, the CBG upgraded its electronic book-entry system in September 2005, and in April 2006 introduced a primary dealership system and shortened the settlement period from five days to one day after the auction date.

25. The CBG has been in a weak financial position for several years, a situation that may constrain its operational independence. It expects to turn a profit in 2006. However, since 2001, it has incurred operational losses ranging from about 2 percent of GDP in 2003 to 0.2 percent in 2005. These losses reflect a combination of factors including the following:

(i) a rundown of international reserves associated with a special loan to the government during 2001-02 at low interest and with a long grace period; (ii) acquisition of other significant non-interest bearing assets (e.g., overdrafts on the governments Treasury Main Account); (iii) sharp increases in foreign currency denominated costs during the period of marked depreciation of the dalasi (2001-03); (iv) large administrative costs; and (v) provisions for various credit losses, some of which reflect loans extended to private enterprises. 
26. In order to strengthen the CBG's operational independence, the government and the CBG have undertaken to ensure compliance with two key provisions of the CBG Act (2005) related to CBG capital and limits on lending to the government. The government will recapitalize the $\mathrm{CBG}$ to the tune of D100 million (the amount stipulated in the CBG Act) over a five year period beginning in 2006. The government has already paid the 2006 contribution of D20 million and has appropriated another D20 million in the 2007 budget.

27. The 2005 CBG Act stipulates that the CBG's lending to the government should not exceed 10 percent of government tax revenues in the previous year. The CBG's claims on the government have exceeded this limit throughout 2006. Since June 2006, the government has acted to reduce substantially the level of its outstanding borrowing from the CBG. The government and the $\mathrm{CBG}$ have undertaken to bring government borrowing from the $\mathrm{CBG}$ in line with the statutory limit by the end of 2007. An action plan indicating how this will be achieved has been provided to IMF staff. The action plan will include a clarification of items covered by the quantitative limit and will also establish a mechanism for ensuring that duration limits on CBG advances to government stipulated in the law are respected.

28. The CBG will continue implementing the Action Plan aimed at strengthening internal controls that was approved by the CBG Board in 2005. In particular, it will make operational the segregation of duties in foreign reserves management as stipulated in the CBG's Policy Guidelines on Segregation of Duties of Foreign Exchange Market Operations.

29. The CBG intends to adopt International Financial Reporting Standards (IFRS) as its accounting framework. As first steps in that direction, it will commission a gap analysis and formulate an Action Plan to convert to IFRS over three years commencing with a gap analysis for the 2006 financial statements. The CBG is also undertaking reforms aimed at strengthening monetary operations and banking supervision. It will also continue work on its research agenda aimed at enhancing the effectiveness of monetary policy.

\section{Financial intermediation}

30. The banking system in The Gambia is relatively sound, but financial intermediation remains low. Profitability and liquidity ratios are high, and the proportion of loans that are nonperforming is relatively small at 13 percent in September 2006. However, the loan-todeposit ratio is very low (40-45 percent) suggesting scope for increased lending activities. In order to facilitate the lending process, the CBG has decided to establish a credit reference bureau. Commercial banks are supportive of the plan and are willing to share the cost of implementing it.

\section{External policies and reforms to enhance external competitiveness}

31. The government is in the process of establishing a business park under the World Bank supported Gateway Project. The project is designed to promote private investment in export-oriented production and employment. The Gambia Investment Promotion and Free Zones Agency (GIPFZA) which was set up under the Gateway Project is fully operational and is actively involved in investment promotion and facilitation. The government is in the process of reviewing the investment act and associated incentive regime. 
32. The World Bank is also helping the government with several studies that should provide material for formulating an action plan to improve the investment climate in The Gambia. The studies include an Investment Climate Assessment and a Diagnostic Trade Integration Study (DTIS). The DTIS is the first stage of The Gambia's participation in the Integrated Framework for Trade-Related Technical Assistance which is designed to help the country integrate more effectively into the regional and global economy through trade. The DTIS draft report which is due in February 2007 will identify key constraints to the expansion and diversification of exports. The final report will contain an Action Plan that will include policy reforms to be undertaken by government and investment projects to be funded by donors.

\section{Other structural reforms}

33. The government has agreed to the privatization of public industrial assets in the groundnut sector, in line with commitments entered into with its bilateral and multilateral donor partners. However, the problems that have beset marketing operations in the sector during recent crop seasons and which have resulted in poor quality exports have undermined confidence in the viability of the sector by local banks and international buyers. A recent World Bank mission that examined the sector concluded that it was still capable of contributing significantly to poverty reduction if the privatization process is professionally implemented in phases. In particular, the mission cautioned that it was necessary to regain the confidence of potential strategic partners before embarking on a full privatization in order to avoid pitfalls experienced with similar ventures in the sub-region. The government is seeking assistance from the EU to commission a study that will map out a privatization strategy for the sector.

34. In view of the importance of capacity constraints in the implementation of government programs, the government is seeking assistance from the World Bank to undertake a comprehensive civil service reform.

\section{Statistics}

35. The quality and timeliness of economic statistics need improvement. Weaknesses in a broad range of economic statistics are hampering the analyses of economic developments in the country. The National Assembly passed a new statistics act in December 2005 that formed the basis for transforming the Central Statistics Department (CSD) into an autonomous Gambia Bureau of Statistics (GBS). The GBS is receiving assistance from the World Bank to use the results of the household budget survey conducted in 2003 to improve the quality of the consumer price index and national accounts statistics. In the area of balance of payments statistics, the CBG has begun to prepare estimates according to the fifth edition of the Balance of Payments Manual.

\section{Prior actions And Program Monitoring}

36. The following actions have been implemented prior to IMF Executive Board consideration of The Gambia's request for a new PRGF arrangement: (i) approval of a budget for 2007 by the National Assembly that is in line with understandings reached 
between representatives of the government and IMF staff on the size of the fiscal basic balance; (ii) submission of government accounts for 2003 to the Auditor-General; and (iii) formulation of an action plan to bring government borrowing from the CBG in line with the limit under the CBG Act (2005) by the end of 2007.

37. Performance under the program will be monitored through a set of quantitative financial targets (Table 1), a set of structural performance criteria and benchmarks (Table 2), and program reviews. The quantitative financial targets for end-March 2007 and endSeptember 2007 are performance criteria, and those for June 2007, and December 2007 are indicative targets. The first and second program reviews are scheduled to be completed by end-July 2007 and end-January 2008, respectively. Definitions of all targeted variables and reporting requirements are contained in the attached Technical Memorandum of Understanding. 


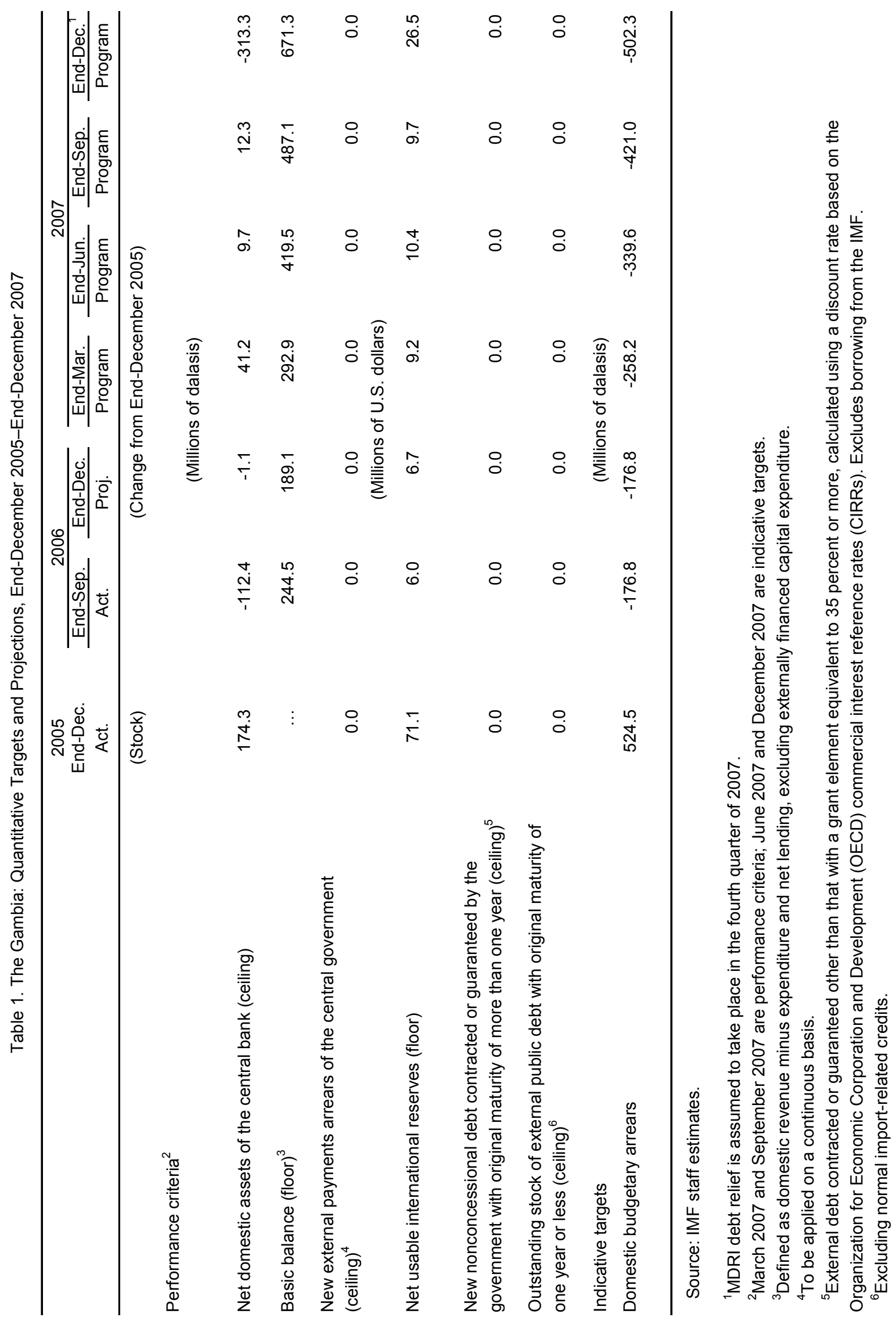


Table 2. The Gambia: Structural Conditionality for December 2006-December $2007^{1}$

\begin{tabular}{|c|c|}
\hline Measure & Target Date \\
\hline \multicolumn{2}{|l|}{ Public financial management and accountability } \\
\hline \multicolumn{2}{|l|}{ 1. Clear the backlog of unaudited government accounts } \\
\hline (i) Submit to the Auditor-General the accounts for 2003 (PA) & End-December 2006 \\
\hline (ii) Submit to the Auditor-General the accounts for 2004 (PC) & End-February 2007 \\
\hline (iii) Submit to the Auditor-General the accounts for 2005 (PC) & End-July 2007 \\
\hline (iv) Submit to the national assembly the audited accounts for $2000-04$ (B) & End-December 2007 \\
\hline (iv) Submit to the Auditor-General the accounts for 2006 (B) & End-December 2007 \\
\hline 2. Make operational the central project management and aid coordination directorate at DoSFEA (PC) & End-June 2007 \\
\hline 3. Launch IFMIS by mid-January 2007 (B) & Mid-January 2007 \\
\hline 4. Establish a comprehensive commitment control system (PC) & End-April 2007 \\
\hline $\begin{array}{l}\text { 5. Prepare and issue a comprehensive monthly budget execution report, including information on } \\
\text { commitments, with a one-month lag, starting with the report for April } 2007 \text { (PC) }\end{array}$ & From May 2007 \\
\hline \multicolumn{2}{|l|}{ Central bank governance and operational independence } \\
\hline $\begin{array}{l}\text { 6. Prepare an action plan to bring government borrowing from the } C B G \text { in line with the } \\
\text { limit under the CBG Act within one year (PA) }\end{array}$ & January 15, 2007 \\
\hline 7. Implement segregation of duties in the management of international reserves (PC) & End-March 2007 \\
\hline \multicolumn{2}{|l|}{ 8. Provide to Fund staff special audit reports on monetary program data at program test dates (PC) } \\
\hline (i) March 2007 report & End-June 2007 \\
\hline (ii) September 2007 report & End-December 2007 \\
\hline \multicolumn{2}{|l|}{ Financial deepening } \\
\hline 9. Establish and staff the credit reference bureau (B) & End-May 2007 \\
\hline \multicolumn{2}{|l|}{ Statistics } \\
\hline 10. Start publishing monthly CPI series based on 2003 household survey weights (PC) & End-June 2007 \\
\hline $\begin{array}{l}\text { 11. Prepare and publish quarterly balance of payments statistics, with a one quarter lag beginning with } \\
2007 \text { Q1 (B) }\end{array}$ & From end-June 2007 \\
\hline
\end{tabular}

${ }^{1} \mathrm{PA}, \mathrm{PC}$, and $\mathrm{B}$ denote prior action, performance criterion, and benchmark, respectively. 


\section{ATTACHMENT II}

\section{THE GAMBIA}

\section{Technical Memorandum of Understanding}

(December 2006-December 2007)

\section{INTRODUCTION}

1. This memorandum sets out the understandings between the Gambian authorities and staff of the International Monetary Fund (IMF) regarding the definitions of quantitative performance criteria, indicative targets, structural performance criteria, and structural benchmarks that will be used in monitoring the Poverty Reduction and Growth Facility (PRGF)-supported program covering the period of 2007-09. It also sets out the related reporting requirements.

\section{Quantitative Performance Criteria}

\section{A. Net Domestic Assets of the Central Bank}

2. Definition. The net domestic assets of the $\mathrm{CBG}$ are defined as the difference between reserve money and the net foreign assets of the $\mathrm{CBG}$. Reserve money is defined as the sum of currency issued by the CBG (i.e., currency in circulation) and the deposits of commercial banks at the CBG. Net foreign assets are defined as foreign assets minus foreign liabilities. Foreign assets and foreign liabilities are defined as claims on nonresidents and liabilities to nonresidents, respectively.

3. For program monitoring purposes, in the calculation of the net domestic assets of the CBG, foreign assets and liabilities will be converted first into U.S. dollars at the prevailing cross-rates and then converted into dalasi using the D/USD program exchange rate. The endof quarter dalasi/U.S. dollar program exchange rates are as follows: 28.10 (December 2006); 28.17 (March 2007); 28.23 (June 2007); 28.30 (September 2007); and 28.36

(December 2007). These are accounting exchange rates only, and should not be construed as projections.

4. Supporting material. Net domestic assets of the central bank will be transmitted as part of the balance sheet of the CBG on a monthly basis within four weeks of the end of each month.

\section{B. Basic Balance of the Central Government}

5. Definition. The basic balance of the central government is defined as revenue (tax and nontax) minus total expenditure and net lending, excluding externally financed capital 
expenditure. Central government excludes local and regional governments and public enterprises.

6. Supporting material. Reporting on the basic balance will form part of the consolidated budget report described in paragraph 26 below.

\section{External Payments Arrears of the Central Government}

7. Definition. External payments arrears are defined as the stock of external arrears on loans contracted or guaranteed by the central government, except on debts subject to rescheduling or a stock of debt operation. They occur when undisputed interest and amortization payments on the above-referenced loans are not made within the terms of the debt contract or in conformity with the terms for interim relief provided under the enhanced HIPC Initiative. This performance criterion will be assessed on a continuous basis.

8. Supporting material. An accounting of nonreschedulable external arrears (if any) by creditor countries, with detailed explanations, will be transmitted on a monthly basis within four weeks of the end of each month. This accounting would include, separately, arrears owed by the central government and other public sector entities to Paris Club and non-ParisClub creditors.

\section{Net Usable International Reserves of the Central Bank of The Gambia}

9. Definition. Net usable international reserves (NIR) of the $\mathrm{CBG}$ are defined as the difference between usable reserve assets and reserve liabilities. Usable reserve assets are readily available claims on nonresidents denominated in foreign convertible currencies. They include the CBG holdings of SDRs, foreign currency cash, foreign currency securities, deposits abroad, and the country's reserve position at the IMF. Excluded are any assets that are pledged, collateralized, or otherwise encumbered, claims on residents, claims in foreign exchange arising from derivatives in foreign currencies vis-à-vis domestic currency (such as futures, forwards, swaps, and options), precious metals, assets in nonconvertible currencies, and illiquid assets (including capital shares in international organizations). Reserve liabilities are all foreign exchange liabilities to residents and nonresidents, including commitments to sell foreign exchange arising from derivatives (such as futures, forwards, swaps, and options), and all credit outstanding from the IMF.

10. Supporting material. End-month data on net usable international reserves of the CBG will be transmitted within seven days of the end of each month.

\section{E. New Nonconcessional Debt Contracted or Guaranteed by the Central Government with Original Maturity of More Than one Year}

11. Definition. This target refers to new nonconcessional external debt with original maturity of more than one year contracted or guaranteed by the central government. It applies 
not only to debt as defined in point No. 9 of the Guidelines on Performance Criteria with Respect to Foreign Debt, adopted by the Executive Board of the IMF on August 24, 2000 (Decision No. 12274-00/85), but also to commitments contracted or guaranteed for which value has not been received. Excluded from this target are loans or purchases from the IMF and debts with a grant element of at least 35 percent. ${ }^{11}$

12. Supporting material. A comprehensive record, including a loan-by-loan accounting of all new concessional and nonconcessional debt contracted or guaranteed by the central government with detailed explanations, will be transmitted on a quarterly basis within four weeks of the end of each quarter. Nonconcessional external debt over one year includes financial leases and other instruments giving rise to external liabilities, contingent or otherwise, on nonconcessional terms.

\section{F. Outstanding Stock of External Public Debt with Original Maturity of One Year or Less}

13. Definition. This target refers to the stock of outstanding external public sector debt with original maturity of one year or less, owed or guaranteed by the central government. ${ }^{12}$ Public sector consists of the central and regional governments and other public agencies, including the CBG. Excluded from this target are normal import-related credits.

14. Supporting material. A comprehensive record of all external debt with original maturity of less than one year owed or contracted by the public sector, with detailed explanations, will be transmitted on a quarterly basis within four weeks of the end of each quarter.

\section{QUANTITATIVE IndiCATIVE TARgets}

\section{A. Domestic Budgetary Arrears}

15. Definition. Domestic budgetary arrears are defined as the sum of all bills that have been received by a central government spending unit or line ministry under the recurrent expenditure budget (including rents and utilities) or the development expenditure budget, and

\footnotetext{
${ }^{11}$ A loan is concessional if its grant element is at least 35 percent, calculated on the basis of the commercial interest reference rates (CIRR) and following the methodology set out in staff paper on Limits on External Debt or Borrowing in Fund Arrangements - Proposed Change in Implementation of the Revised Guidelines approved by the IMF Executive Board on April 15, 1996.

12 The term "debt" has the meaning set forth in point No. 9 of the Guidelines on Performance Criteria with Respect to Foreign Debt, adopted by the Executive Board of the IMF on August 24, 2000 (Decision No. 12274-00/85).
} 
for which payment has not been made within 30 days. Arrears can be cleared in cash or through debt swaps.

16. Supporting material. A comprehensive record of all domestic budgetary arrears, with detailed explanations, will be transmitted on a quarterly basis within four weeks of the end of each quarter.

\section{Structural Performance Criteria and Benchmarks}

\section{A. Make the Central Project Management and Aid Coordination Directorate Operational}

17. Definition. The directorate is deemed operational when it is fully staffed and it begins establishing a database on external aid received by all departments of government.

18. Supporting material. A comprehensive record of external aid received by all departments of government, by donor and project, will be transmitted on a quarterly basis within four weeks of the end of each quarter.

\section{B. Commitment Control System}

19. Definition. The target on establishing a comprehensive system will be deemed to be met if IFMIS becomes operational and is used to track expenditure commitments made by all government departments and agencies covered by the central government budget. In the event IFMIS is delayed, the target will be deemed to be met if the manual commitment control system introduced on a pilot basis in 2006 is extended to all government departments and agencies covered by the central government budget.

20. Supporting material. A monthly budgetary execution report with detailed information on expenditure appropriations, commitments, payment orders, and actual payments by broad category of spending will be transmitted within four weeks of the end of each month. The coverage of the monthly budget execution report will include all government departments and agencies covered by the central government budget.

\section{Segregation of Duties in the Management of International Reserves}

21. Definition. This target refers to the item in the CBG's "Policy Guidelines on Segregation of Duties of Foreign Exchange Market Operations" which prescribes that front and back office responsibilities will be assigned to the Foreign and the Finance Departments, respectively, and which envisages the establishment of a middle office.

22. Supporting material. The CBG's Internal Audit Department will issue a report on compliance with the Guidelines. 


\section{Special Audit Reports on Monetary Program Data}

23. The special audits will be conducted on the basis of the draft terms of reference dated December 19, 2006, agreed between CBG and IMF staffs.

\section{Other DATA REQuirements ANd REPORTING STANDARDS}

24. In addition to providing the data needed to monitor program implementation in relation to the program's performance criteria, indicative targets, and benchmarks, as set out above, the authorities will transmit the following data within the time frame specified below:

\section{A. Prices}

25. The monthly disaggregated consumer price index will be transmitted within four weeks of the end of each month.

\section{B. Government Accounts Data}

26. The following monthly reports will be transmitted to the IMF within two weeks of the end of the month: (i) revenue flash report; and (ii) expenditure flash report.

27. A monthly consolidated central government budget report (i.e., the analytical table) on budget execution during the month and cumulatively from the beginning of the year, will be transmitted to the IMF within four weeks of the end of the month. The report will comprise: (i) revenue data by major item, including tax (direct tax, taxes on domestic goods and services, and taxes on international trade) and nontax; (ii) external grants by type (e.g., project, program); (iii) details of recurrent expenditure (including data on wages and salaries, interest payments, and other charges); (iv) details of capital expenditure and net lending (including data on externally financed capital expenditure, expenditures from the Gambia Local Fund, and net lending); (v) the overall balance and the basic balance (defined in paragraph 5); (vi) details of budget financing (including net domestic borrowing and its gross components, external grants, net external borrowing and its gross components, utilization of privatization proceeds, and arrears).

28. Net domestic borrowing by the central government over a given period is defined as the change in the net domestic debt at the end of the period minus the net domestic debt at the beginning of the period. The central government's net domestic debt is defined as: claims on the central government by the banking system minus deposits of the central government with the banking system plus claims by the nonbanking sector, including public enterprises. Central government excludes local and regional governments and public enterprises. The banking system comprises the Central Bank of The Gambia (CBG) and commercial banks. 


\section{Poverty Reducing Expenditure}

29. A monthly report on poverty-reducing expenditures, by functional and economic classifications, will be transmitted within four weeks of the end of each month. Poverty reducing expenditures comprise line items in the budget that have been specifically tagged as PRSP-related. For 2007, they include expenditure on the construction of trunk roads.

\section{Financial Information on Selected Public Enterprises}

30. Quarterly financial data in the format of Annex I will be transmitted to the IMF within four weeks of the end of the quarter.

\section{E. Monetary Sector Data}

31. The balance sheets of the CBG, prepared on the bases of current and program exchange rates, will be transmitted on a monthly basis to the IMF within four weeks of the end of each month. The balance sheet should explicitly identify all claims on government. In addition, balances in the treasury main, sterilization, and other deposit accounts should also be presented. The consolidated balance sheet of the commercial banks and a monetary survey (i.e., consolidation of the accounts of the $\mathrm{CBG}$ and the commercial banks) will be transmitted within six weeks of the end of each month.

\section{F. Treasury Bills and CBG Bills}

32. Weekly data on the issuance and outstanding stocks of treasury bills and CBG bills, and on the yields (interest rates) of the various instruments will be transmitted on a monthly basis within seven days of the end of the month. The weekly Liquidity Management Reports (LMRs) will be transmitted on a weekly basis within seven days of the end of the week. Data on treasury bills outstanding (including information on the distribution by bank and nonbank holders) will be transmitted on a monthly basis within six weeks of the end of each month. The monthly LMR, reflecting the data as of the last working day of the month, will be transmitted within seven days after the end of each month.

\section{G. External Sector Data}

33. The following standards will be adhered to in reporting data on exchange rates: (i) the interbank market exchange rates, defined as the simple average of the weekly weighted average buying and selling rates, will be transmitted on a weekly basis within five business days of the end of the week; and (ii) the CBG's published monthly average and end-month exchange rates, including those for all currencies in which foreign assets and liabilities are denominated, will be transmitted within two weeks of the end of the month. 
34. The CBG will compile balance of payments statistics on a quarterly basis beginning from the first quarter of 2007. The balance of payments data will be transmitted to the IMF with a one quarter lag. 
ANNEX I

\section{QuARTERly Reporting System FOR PUblic ENTERPRISES}

Name of the Enterprise:

Supervisory Authority:

Regulatory Authority (if any):

Law Establishing the Company:

Government Shares (percent of total capital):

Date of Last Audited Accounts:

\begin{tabular}{|c|c|c|c|c|}
\hline Fiscal Year & First Quarter & Second Quarter & Third Quarter & Fourth Quarter \\
\hline \multicolumn{5}{|l|}{ Revenue } \\
\hline \multicolumn{5}{|l|}{ (Cost of sales) } \\
\hline \multicolumn{5}{|l|}{ Gross profit } \\
\hline \multicolumn{5}{|l|}{$\begin{array}{l}\text { (Administrative } \\
\text { expenditures) }\end{array}$} \\
\hline \multicolumn{5}{|l|}{ (Depreciation) } \\
\hline \multicolumn{5}{|c|}{ Net financing income/cost } \\
\hline \multicolumn{5}{|c|}{ Net operating profit } \\
\hline \multicolumn{5}{|c|}{ Memorandum items: } \\
\hline \multicolumn{5}{|l|}{ Taxes paid } \\
\hline \multicolumn{5}{|l|}{ - Sales tax } \\
\hline \multicolumn{5}{|l|}{ - Income tax } \\
\hline \multicolumn{5}{|c|}{ - International trade } \\
\hline \multirow{2}{*}{\multicolumn{5}{|c|}{$\begin{array}{l}\text { Other taxes } \\
\text { Dividends to government }\end{array}$}} \\
\hline & & & \multirow{2}{*}{\multicolumn{2}{|c|}{ Dividends to government }} \\
\hline \multicolumn{4}{|c|}{ Transfers received } & \\
\hline \multicolumn{5}{|c|}{ - From government } \\
\hline \multirow{2}{*}{\multicolumn{5}{|c|}{$\begin{array}{c}\text { - Other } \\
\text { Stock of debt }\end{array}$}} \\
\hline \multirow{2}{*}{\multicolumn{5}{|c|}{ Stock of debt }} \\
\hline & & & & \\
\hline \multirow{2}{*}{\multicolumn{5}{|c|}{$\begin{array}{l}\text { - Banking system } \\
\text { New investment }\end{array}$}} \\
\hline \multirow{2}{*}{\multicolumn{5}{|c|}{$\begin{array}{l}\text { New investment } \\
\text { Available cash }\end{array}$}} \\
\hline & & & & \\
\hline \multicolumn{5}{|l|}{ - In banks } \\
\hline - Other & & & & \\
\hline Stock of arrears & & & & \\
\hline
\end{tabular}




\title{
INTERNATIONAL MONETARY FUND
}

\section{THE GAMBIA}

\section{Request for a Three-Year Arrangement Under the Poverty}

Reduction and Growth Facility and for Additional Interim Assistance Under the Enhanced Initiative for Heavily Indebted Poor Countries-Informational Annex

\author{
Prepared by the African Department \\ (In collaboration with other departments) \\ Approved by Michael Nowak and Adrienne Cheasty
}

February 7, 2007

- Relations with the Fund. Describes financial and technical assistance from the Fund and provides information on the safeguards assessment and exchange rate system. Outstanding purchases and loans amounted to SDR 11.82 million (38.01 percent of quota) at end-December 2006.

- $\quad$ Relations with the World Bank. Describes the World Bank Group program and portfolio, including a statement of IFC investments.

- $\quad$ Relations with the African Development Bank. Describes the African Development Bank Group program and portfolio.

- $\quad$ Statistical Issues. Assesses the quality of statistical data. Weaknesses in a broad range of economic statistics are hampering the analyses of economic developments in the country. 


\section{The Gambia: Relations with the Fund}

(As of December 31, 2006)

Membership status. Joined September 21, 1967. The Gambia accepted the obligations under Article VIII, Sections 2(a), 3, and 4, of the Fund's Articles of Agreements on January 21, 1993. It maintains an exchange system that is free of restrictions on the making of payments and transfers for current international transactions.

General Resources Account

Quota

Fund holdings of currency

Reserve position in Fund

SDR Department

Net cumulative allocation

Holdings

Outstanding Purchases and Loans

Poverty Reduction and Growth Facility (PRGF)

arrangements

\begin{tabular}{|c|c|}
\hline$\underline{\text { SDR Million }}$ & $\%$ Quota \\
\hline 31.10 & 100.0 \\
\hline 29.62 & 95.23 \\
\hline 1.48 & 4.77 \\
\hline
\end{tabular}

\begin{tabular}{cc} 
SDR Million & \% Allocation \\
\hline 5.12 & 100.0 \\
0.97 & 19.01
\end{tabular}

$\underline{\text { SDR Million }}$

11.82

\% Quota

38.01

\section{Latest Financial Arrangements}

$\begin{array}{lcccc}\text { Type } & \text { Approval Date } & \text { Expiration Date } & \begin{array}{c}\text { Amount } \\ \text { Approved } \\ \text { (SDR Million) }\end{array} & \begin{array}{c}\text { Amount Drawn } \\ \text { (SDR Million) }\end{array} \\ \text { PRGF } & \text { Jul. 18, 2002 } & \text { Jul. 17, 2005 } & 20.22 & 2.89 \\ \text { PRGF } & \text { Jun. 29, 1998 } & \text { Dec. 31, 2001 } & 20.61 & 20.61 \\ \text { PRGF } & \text { Nov. 23, 1988 } & \text { Nov. 25, 1991 } & 20.52 & 18.02\end{array}$

Projected Obligations to the Fund (SDR million; based on current use of resources and present holdings of SDRs)

Principal

Charges/interest

Total

\begin{tabular}{lllll}
\multicolumn{5}{c}{ Forthcoming } \\
\hline$\underline{2007}$ & $\underline{2008}$ & $\underline{2009}$ & $\underline{2010}$ & $\underline{2011}$ \\
2.75 & 3.33 & 2.64 & 1.95 & 0.58 \\
$\underline{0.23}$ & $\underline{0.21}$ & $\underline{0.19}$ & $\underline{0.18}$ & $\underline{0.17}$ \\
$\underline{2.97}$ & $\underline{3.53}$ & $\underline{2.83}$ & $\underline{2.13}$ & $\underline{0.75}$
\end{tabular}




\section{Implementation of HIPC Initiative}

$\begin{array}{lc}\text { Commitment of HIPC assistance } & \begin{array}{c}\text { Enhanced } \\ \text { Framework }\end{array} \\ & \\ \text { Decision point date }^{1} & \text { Dec. 11, 2000 } \\ \text { Assistance committed (yearend } 2000 \mathrm{NPV} \text { terms) }{ }^{2} & \\ \text { Total assistance (US\$ million) } & \\ \text { Of which: IMF assistance (US\$ million) } & 66.60 \\ \text { SDR equivalent, million } & 2.30 \\ \text { Completion point date } & 1.80 \\ & \text { Floating } \\ \text { Disbursement of IMF assistance (SDR million) } & \\ \text { Assistance disbursed } & \\ \quad \text { Interim assistance } & 0.08 \\ \quad \text { Completion point balance } & 0.08 \\ \text { Additional disbursement of interest income } & \ldots\end{array}$

\section{Safeguards assessments}

A FIN Safeguards Assessment mission visited The Gambia in March 2003 and in November 2003 to conduct a Stage 1 onsite assessment of the Central Bank of The Gambia (CBG). The assessment was completed on February 3, 2004. FIN conducted an update Safeguards Assessment of the CBG in November 2006. This update assessment was performed against the backdrop of previous safeguards findings, which identified significant vulnerabilities in the CBG's safeguards framework. ${ }^{4}$ While the CBG has instituted a series of control reforms since that assessment, a key challenge looking forward is to build on existing progress to strengthen remaining vulnerabilities in the CBG's safeguards framework, including controls over international reserves and procedures for extending credit to the

\footnotetext{
${ }^{1}$ The Fund approved the decision on 12/15/2000 as Decision 12365-(00/126). The World Bank Board decision was taken on $12 / 14 / 2000$.

2 Assistance committed under the enhanced HIPC Initiative is expressed in net present value (NPV) terms at the decision point.

${ }^{3}$ Under the enhanced HIPC Initiative, an additional disbursement is made at the completion point corresponding to interest income earned on the amount committed at the decision point but not disbursed during the interim.

${ }^{4}$ A summary of its findings and recommendations is outlined in Box 3 of the 2003 Article IV consultation report (see IMF Country Report No. 04/143 of May 2004).
} 
government. The update assessment recommended certain priority actions be taken, including semi-annual audits of monetary program data, the implementation of segregation of duties in the reserves management function, a phased implementation of International Financial Reporting Standards, and an action plan to ensure that government borrowing from the CBG is brought within the statutory limits.

\section{Exchange rate arrangement}

Until January 20, 1986, the Gambian currency, the dalasi, was pegged to the pound sterling at a rate of D5 $=£ 1$. On January 20,1986 , an interbank market for foreign exchange was introduced, and since then the exchange rate has been determined by market forces. Effective June 30, 2002, the exchange rate arrangement of The Gambia was reclassified from independently floating to managed float with no preannounced path. As of December 31, 2006, the midpoint exchange rate in the interbank market was D28.0469 per U.S. dollar.

The Gambia has been part of the exchange rate mechanism of the West African Monetary Zone (WAMZ) since April 2002.

\section{Last Article IV consultation}

The Executive Board concluded the 2006 Article IV consultation on October 13, 2006 (see IMF Country Report No. 06/444 of December 2006).

\section{Technical assistance}

The Fund has been providing The Gambia with extensive technical assistance in macroeconomic, fiscal, and monetary areas, and in improving the compilation of macroeconomic statistics. Specific technical assistance projects are the following:

\section{Fiscal Affairs Department}

Sep. 2004-May 2006

Peripatetic regional advisor assisted the authorities in putting the new organic budget law into effect, strengthening public expenditure management, and improving the reporting of budget execution.

Feb./Mar. 2004

Mission worked jointly with the World Bank on the Assessment and Action Plan (AAP).

Apr. 2003

TA advisor reviewed reforms in public expenditure management.

Mar. 2003-Oct. 2003

Long-term resident budget expert helped the authorities to strengthen budgetary expenditure reporting and control.

Dec. 2002

TA advisor advised the authorities on drafting an organic budget bill. 
Oct. 2002

Mar. 2002-Oct. 2003

Nov. 2001-Oct. 2003

Jul. 2001

Aug. 2000-Aug. 2001

Sep. 1999

Jan./Feb. 1996
TA advisor reviewed reforms in public expenditure management. Long-term resident budget expert helped the authorities to strengthen budgetary expenditure reporting and control, initially for a year beginning from March 2002. The assignment was later extended until October 2003.

Peripatetic advisor assisted the DoSFEA in revenue administration reforms, including customs, implementing a large-taxpayer unit, and establishing a central revenue authority.

TA mission assessed the authorities' capacity to track povertyrelated spending.

Long-term resident budget expert assisted the authorities in strengthening budgetary expenditure reporting and control.

TA mission assisted the authorities in expenditure management, budget execution issues, cash and debt management, short-term financial planning, fiscal reporting, and information systems.

Joint FAD/United Nations Development Program (UNDP) TA mission helped set up a system for monitoring the financial operations of public enterprises.

\section{Monetary and Capital Markets Department/ Monetary and Financial Systems Department /Monetary and Exchange Affairs Department}

Jul./Aug. 2006

Apr./May 2006

Apr./May 2006

Nov. 2005

Mar. 2005

Oct. 2004

Jul. 2002
Mission reviewed progress made in strengthening the CBG's capacity in monetary operations and liquidity forecasting, foreign exchange operations, and foreign reserves management.

Technical expert advised the CBG on banking supervision.

Technical expert advised the CBG on improving monetary operations.

Technical expert advised the CBG on improving monetary operations.

Follow-up to the October 2004 mission.

Advisory mission made recommendations for improving monetary and foreign exchange operations and for reorganizing the central bank.

TA diagnostic mission focused on financial supervision and the insurance sector; and helped review the Central Bank Act and draft the Financial Institutions and Insurance Act. 
Dec. 2001

May 2001

Apr. 2001

May 2000

Nov. 1999

Aug. 1998

Dec. 1996

Jan./Feb. 1994

\section{Statistics Department}

Apr./May 2006

Feb. 2006

Feb. 2005

$2002-04$
TA diagnostic mission focused on strengthening CBG ability to , formulate and implement monetary policy and manage its foreign exchange operations and the financial system.

Short-term expert helped the authorities to design operational, prudential, and policy safeguards (including assessing the adequacy of legislation) for introducing foreign-currencydenominated accounts.

Short-term expert helped the authorities to set up a book-entry system.

Short-term expert helped the authorities to set up a short-term liquidity forecasting system.

Short-term expert helped the authorities design operational, prudential, and policy safeguards (including assessing the adequacy of legislation) for introducing foreign-currencydenominated accounts in the banking system.

TA mission helped the CBG draft market-based monetary policy instruments and review its program for strengthening banking supervision.

Technical expert helped the CBG in foreign exchange operations.

TA mission worked on monetary management and bank supervision.

TA mission helped to improve the compilation and analytical soundness of monetary and financial statistics.

TA mission advised on compilation of balance of payments statistics.

Report on the Observance of Standards and Codes (ROSC) Data Module - mission assessed data quality in four main areas of macroeconomic statistics (national accounts, government finance, monetary, and balance of payments) based on the Fund's Data Quality Assessment Framework (DQAF, July 2003) and The Gambia's dissemination practices against the recommendations of the General Data Dissemination System (GDDS).

Peripatetic statistical advisor helped the Central Statistics Department update CPI data and improve the compilation of national accounts statistics. 
May 2003

Aug. 2001

Sep. 2000

Nov. 1999

Jun./Jul. 1999

Nov./Dec. 1998

\section{Others}

Apr. 2002-Apr. 2004
Mission advised on building an integrated database to report monetary statistics for all IMF data submissions.

TA mission advised on compilation of monetary and financial statistics.

TA mission advised on compilation of balance of payments statistics.

Mission reviewed collection of statistics to develop GDDS metadata for The Gambia.

TA mission advised on compilation of balance of payments statistics.

TA mission advised on national accounts statistics.

A long-term resident macroeconomic advisor was assigned initially for a year beginning from April 2002, and later the assignment was extended by one more year through April 2004.

\section{Resident Representative}

Mr. Alex Segura-Ubiergo was appointed the Fund's first Resident Representative to The Gambia in September 2006. He is also the Resident Representative to Senegal and is based in Dakar. 


\section{The Gambia: Relations with the World Bank Group}

(As of January 5, 2007)

The Bank and the Fund cooperate closely in providing support for the implementation of The Gambia's development strategy. The country's strategy is set forth in the Poverty Reduction Strategy Paper (PRSP), called the "Strategy for Poverty Alleviation II", which was finalized in April 2002 and endorsed by the Boards of the World Bank and IMF in July 2002. It outlines a medium-term development strategy to reduce poverty through the following five key objectives: (1) macroeconomic stability and effective public resource management; (2) promotion of pro-poor growth and employment through private sector development, particularly in the rural agricultural sector; (3) improved basic social services and infrastructure; (4) capacity building of local communities and civil society organizations (CSOs); and (5) strengthened multisectoral programs to reduce population growth, gender inequality, HIV/AIDS, malnutrition, and environmental degradation. The PRSP includes a multidimensional poverty analysis, an outline of a monitoring and evaluation framework, preliminary performance indicators and targets, estimates of the cost of implementation, and an indicative financing gap. The PRSP covered the period from 2003 to 2005. The authorities are in the final stages of preparing the second PRSP. The new PRSP covers 2007 to 2011.

The Bank's support to The Gambia is outlined in its Country Assistance Strategy (CAS). The CAS is aligned with the PRSP, particularly in the following areas: (1) establishing a macroeconomic and sectoral environment conducive to economic growth; (2) rehabilitating and building infrastructure; (3) enhancing human capital by providing more efficient social services; and (4) building capacity in departments responsible for economic management. The Bank's direct support in recent years has mainly been sector-based investment projects and analytical work. The Fund has traditionally led the dialogue on macroeconomic policy, including fiscal, monetary, and exchange rate policies. Areas of close collaboration include public expenditure management reform and government statistics. Collaboration on trade issues should soon increase with the planned Integrated Framework analysis led by the Bank. The next CAS is being prepared in FY07.

As of January 5, 2007, IDA had approved 34 credits worth a total of about US\$296 million. The current portfolio consists of five projects in capacity building for economic management, private sector development (trade gateway), education, community-driven development, and locust prevention (regional), totaling US\$52.9 million, of which US\$33.8 million is undisbursed.

A Capacity Building for Economic Management Project was approved in July 2001 to help build (1) government capacity for economic planning, policy formulation, and execution, and (2) the capacity of the judicial and financial systems to facilitate private sector development. Reforms supported by the project include establishment of the Revenue Authority, implementation of the Integrated Financial Management Information System 
(IFMIS), capacity building and restructuring of the Central Statistics Department (CSD), and establishment of an alternative dispute resolution (ADR) court system.

The Trade Gateway Project was approved in February 2002 to help the country establish itself as a globally competitive business park by laying the foundations for expanded private investment, export-oriented production, and employment through a free zone and an improved institutional environment.

The second phase of the Education Project was approved on June 1, 2006, to help The Gambia improve conditions for teaching and learning in basic education by (1) improving the performance of students, teachers, and schools, (2) strengthening capacity building and performance management, and enhancing monitoring and evaluation, and (3) expanding access to underserved communities.

A Community-Driven Development Project was approved on August 31, 2006, to help rural communities, in partnership with local government authorities, plan, implement and maintain their priority social and economic investments.

A regional Emergency Locust Project covering Burkina Faso, Chad, Mali, Mauritania, Niger, Senegal, and The Gambia was approved in December 2004. To help reduce the hardships imposed on people and the environment by current and future locust invasions, the project supports improved strategies for prevention, early warning, reaction, and mitigation at both the national and regional levels.

In addition to investment projects, the Bank is currently conducting several analytical initiatives, some of them jointly with other donors: (i) civil service reform and capacity assessment; (ii) poverty assessment; (iii) Diagnostic Trade Integrated Framework (DTIS) study; and (iv) Investment Climate Assessment (ICA).

As of July 31, 2006, the IFC's portfolio had three investments in The Gambia with a balance of about US $\$ 0.75$ million. The investments are for a medical clinic (Ndebaan) and commercial fishing (Lyefish).

IDA, MIGA, and the IFC will continue to coordinate their efforts to support development activities in The Gambia. Their activities are enhanced by the IFC office in Dakar, which also oversees IFC activities in The Gambia. 
Summary of IDA Credits in The Gambia

(As of January 5, 2007; millions of U.S. dollars)

\begin{tabular}{lrrr}
\hline Projects & Commitment & Disbursed & \multicolumn{2}{c}{ Undisbursed } \\
\hline & & & \\
Ongoing projects & 12.0 & 0.0 & 12.6 \\
$\quad$ Community-Driven Development & 8.0 & 0.0 & 8.4 \\
Education Phase II & 16.0 & 10.6 & 7.6 \\
Gateway & 15.0 & 13.6 & 3.6 \\
$\quad$ Capacity Building for Economic & 1.9 & 0.3 & 1.6 \\
Management Project & & & 33.8 \\
Africa Emergency Locust & 52.9 & 24.5 & \\
$\quad$ Subtotal & & & \\
\end{tabular}

Source: The World Bank Integrated Controller's System.

The Gambia: IFC's Held and Disbursed Portfolio

(As of July 31, 2006; millions of U.S. dollars)

\begin{tabular}{|c|c|c|c|c|c|c|c|c|c|}
\hline \multirow[b]{3}{*}{ FY Approval } & \multirow[b]{3}{*}{ Company } & \multirow{2}{*}{\multicolumn{3}{|c|}{ Committed/Held }} & \multicolumn{5}{|c|}{ Disbursed } \\
\hline & & & --IFC--. & -- & & --.-- & ---IFC--. & -- & \\
\hline & & Loan & Equity & Quasi & Partic & Loan & Equity & Quasi & Partic \\
\hline 1993 & $\begin{array}{l}\text { AEF Ndebaan } \\
\text { (Medical Clinic) }\end{array}$ & 0.2 & 0.0 & 0.0 & 0.0 & 0.2 & 0.0 & 0.0 & 0.0 \\
\hline 1994 & $\begin{array}{l}\text { AEF Lyefish } \\
\text { (Commercial Fishing) }\end{array}$ & 0.36 & 0.0 & 0.0 & 0.0 & 0.36 & 0.0 & 0.0 & 0.0 \\
\hline \multirow[t]{2}{*}{1997} & KKF II & 0.19 & & & & 0.19 & & & \\
\hline & Total portfolio & 0.75 & 0.0 & 0.0 & 0.0 & 0.75 & 0.0 & 0.0 & 0.0 \\
\hline \multicolumn{2}{|c|}{ Pending commitments } & 0.0 & 0.0 & 0.0 & 0.0 & & & & \\
\hline
\end{tabular}

Source: IFC.

Questions may be referred to Mr. Soh Hoon (email: hsoh@worldbank.org) or Ms. Françoise Perrot (email: fperrot@worldbank.org). 


\section{The Gambia: Relations with the African Development Bank}

(As of November 30, 2006)

The African Development Bank (AfDB) Group began lending to The Gambia in 1974. As of November 30, 2006, it had approved, 53 operations with total commitments, net of cancellations of UA205.2 million (US\$305.5 million) in the following sectors: transport (25 percent); agriculture (21.7 percent); social (24.6 percent); public utilities (12.1 percent); multisectoral ( 8.8 percent); environment (5.8 percent); and industry ( 2.0 percent). ${ }^{5}$ About 84.7 percent of the Bank Group's net commitments were made from the resources of the African Development Fund (ADF), 8.6 percent from the AfDB nonconcessional window, and 6.7 percent from the Nigeria Trust Fund (NTF).

As of November 30, 2006, 35 operations had been completed, 3 were cancelled at the government's request, and 15 others continue, including 3 multinational projects, all in agriculture. Implementation of the portfolio is generally satisfactory; it achieved a rating score of 2.01 (on a scale from 0 to 3 ) during the Bank Group's 2005 portfolio review. The portfolio has a relatively low project-at-risk (PAR) rate of 33.3 percent, which compares well with the Bank-wide average of 43.3 percent stated in the AfDB's 2004 Annual Portfolio Performance Review (APPR). Total disbursement rates for the portfolio are also satisfactory. By the end of August 2006, they were 84.3 percent (overall), 100 percent (AfDB-financed projects), 83.8 percent (ADF), and 44.5 percent (NTF). The satisfactory performance of the portfolio is attributed to the supervision missions conducted by the AfDB, especially since 2003.

The AfDB is also a major participant in The Gambia's enhanced Heavily Indebted Poor Countries (HIPC) Initiative program, under which it is due to grant debt relief of US\$15.7 million in net present value (NPV) terms (23.6 percent of total debt relief under the program).

The AfDB's strategy for The Gambia for the period 2005-09 is still being prepared and is awaiting finalization of the country's new Poverty Reduction Strategy Paper (PRSP). The draft Country Strategy Paper (CSP) proposes to improve productive capacity through microfinance management and credit projects and to enhance economic management. The CSP has yet to be approved. The last CSP for The Gambia, which covered 2002-04, was designed to help the country in its efforts to meet the Millennium Development Goals (MDGs) by addressing specific institutional and human capacity constraints. The plan is based on The Gambia's poverty reduction strategy (SPA-II), and the base-case scenario focuses on the interventions shown in the table below.

\footnotetext{
${ }^{5} \mathrm{UA}$ stands for unit of account $=1 \mathrm{SDR}$ (currently equivalent to about \$1.49).
} 


\begin{tabular}{|c|c|c|}
\hline Objective & $\begin{array}{l}\text { Instrument } \\
\text { and Amount }\end{array}$ & Focus \\
\hline Meet the MDGs & $\begin{array}{l}\text { Third education } \\
\text { project, } \\
\text { UA10.0 million }\end{array}$ & $\begin{array}{l}\text { Increase access to quality education and } \\
\text { skills development, particularly for girls } \\
\text { and pupils in the poorest areas of the } \\
\text { country. }\end{array}$ \\
\hline $\begin{array}{l}\text { Entrepreneurship } \\
\text { Development and } \\
\text { Livelihood } \\
\text { Promotion }\end{array}$ & UA8.0 million & $\begin{array}{l}\text { Enhance entrepreneurial skills and improve } \\
\text { capacity and income generation ability by } \\
\text { providing microfinance. Focus is poverty } \\
\text { reduction. }\end{array}$ \\
\hline $\begin{array}{l}\text { Implement } \\
\text { multisector } \\
\text { capacity building } \\
\text { (planned for ADF- } \\
\text { X lending cycle in } \\
\text { the context of the } \\
\text { CSP being } \\
\text { prepared) }\end{array}$ & $\begin{array}{l}\text { Capacity-building } \\
\text { project, grant of } \\
\text { UA2.0 million }\end{array}$ & $\begin{array}{l}\text { Strengthen capacity of departments and } \\
\text { institutions involved in preparing and } \\
\text { implementing the PRSP/SPA-II. Extend } \\
\text { support to institutions dealing in economic } \\
\text { and political governance, including the } \\
\text { Department of Justice, Auditor General's } \\
\text { Department, Public Divestiture Agency, } \\
\text { parliament, and the Public Procurement } \\
\text { Agency. }\end{array}$ \\
\hline
\end{tabular}

Under ADF-X, The Gambia is a grants-only recipient. Additional resources can be made available if there is improvement in both performance under the Country Policy Institutional Assessment (CPIA) and portfolio performance.

The AfDB's strategy is implemented through both lending and nonlending activities. The extent of lending depends on finalization of the new PRSP, satisfactory performance under the IMF-supported PRGF, progress toward reaching the HIPC Initiative completion point, advances in strengthening public expenditure management, and improved performance of AfDB-sponsored projects. Nonlending intervention is designed to strengthen policy dialogue between the government and stakeholders and focuses mainly on studies, funded through grants, to improve governance, mainstream gender, fight HIV/AIDS and communicable diseases, and improve the energy supply. 


\section{The Gambia: Statistical Issues}

The poor quality of some data and complete lack of others hamper the analysis of economic developments. There are substantial weaknesses in The Gambia's economic and financial statistics, especially in national accounts, balance of payments, and external debt statistics. Data reporting by the authorities to the Fund is somewhat irregular. The Gambia participates in the General Data Dissemination System (GDDS) and its metadata were posted on the Fund's Dissemination Standards Bulletin Board on May 22, 2000 and updated in the second half of 2003.

A data ROSC mission visited The Gambia in February 2005 to assess data quality in four main areas of macroeconomic statistics (national accounts, government finance, monetary, and balance of payments) based on the Fund's Data Quality Assessment Framework (DQAF, July 2003). Data dissemination was also assessed in terms of the GDDS recommendations. The mission recommended establishment of an interim Statistics Council to spearhead passage of the Statistics Act, eventual creation of an autonomous The Gambia Bureau of Statistics (GBOS), and preparation of a comprehensive statistics reform package. Accordingly, the National Assembly passed a new statistics act in December 2005. Work began in June 2006 to implement the plan for transforming the Central Statistics Department (CSD) to GBOS. Recently, Statistics Council and Statistician General have been appointed.

\section{Real sector}

Annual national account estimates are compiled using only the production approach. In the absence of more comprehensive information, they rely heavily on indicators. In response to STA assistance on national accounts, the CSD prepared a program to (1) improve the quality of source statistics by improving and expanding existing surveys; (2) improve the coverage and methodology of GDP estimates, including implementation of the System of National Accounts (1993 SNA); (3) rebase the constant price estimates - the current baseline is 1976/77 - to a more recent period; and (4) make independent estimates of expenditure aggregates. An STA peripatetic adviser helped the authorities to compile national accounts and price statistics.

Currently, the GBOS produces data on the consumer price index (CPI) based only on the 1976/77 weights for the low-income population of the greater Banjul area. The World Bank has been providing technical assistance to the GBOS to update CPI weights using the 2003 household expenditure survey to better reflect current consumption patterns, and update the national accounts and expand the compilation to include independent estimates of gross domestic expenditure. The authorities are making progress in these efforts. As in the case of the national accounts, improvements in the CPI will require that the GBOS have adequate resources. 


\section{Government finance}

The authorities release data on central government transactions with a lag of about eight weeks for both revenue and expenditure. Reporting in the treasury ledger is subject to considerable delay; the central government accounts for 1991-99 were not audited until 2005. Inadequacies persist in compiling data on an economic basis and in tracking foreign-financed expenditure, including HIPC debt relief. Monthly data on domestic government financing are available with a delay of six to eight weeks.

\section{Monetary data}

The CBG has improved its data reporting to the Fund, but sometimes it reports data with delay. The commercial banks follow a uniform chart of accounts to report financial data to the CBG. They also transmit flash reports of key monetary data to the CBG on a weekly basis. Several times over the past few years, the CBG has reclassified the monetary accounts to improve sectorization and coverage, as well as to harmonize the data reported to STA and AFR. In particular, there were substantial revisions to data on foreign exchange transactions and lending to the central government for 2001-03. The monetary and financial statistics mission in April-May 2006 made further recommendations to expand the coverage of depository corporations to include credit unions. The mission also recommended that accrued interest be included in the value of financial instruments. Given the significant influence of the Social Security and Housing Development Corporation in monetary developments, the mission recommended compilation of a financial survey. To improve the accuracy and classification of government accounts, it designed a supplementary form for reporting government positions at the CBG, to be reported to the IMF monthly. It also introduced standardized report forms to make it easier for the authorities to report data to the Fund for the use of both AFR and STA.

\section{Balance of payments}

A significant proportion of The Gambia's external transactions, including reexports, are informal. Including reexport trade in the balance of payments assumes that reexports are a fixed share of total imports. Tourism receipts are derived from a simple function of tourist arrivals and average length of stay in The Gambia. Data on private capital flows are poor but the CBG has taken recent initiatives to improve on them. Official grant and loan disbursements and repayments are relatively well recorded, but there are some gaps in project disbursements. Data on the gross and net CBG international reserves bank are available with a short lag, but had to be substantially revised for 2001-03.

STA provided technical assistance on balance of payment statistics in June-July 1999, September 2000, and February 2006. Until the February 2005 ROSC mission, progress in implementing STA recommendations on balance of payments had been slow. 
On the recommendation of the ROSC mission, the CBG revamped the balance of payments unit by elevating it to a full section with additional staff. To strengthen capacity to compile balance of payments data, the Fund provided substantial training to the CBG staff in 200506. A member of the CBG staff attended STA's Balance of Payments Statistics course in May-June 2005 and another attended in May-June 2006. CBG staff also participated in the regional balance of payments seminar offered by STA in Banjul early in 2006.

In line with the recommendations of the February 2006 balance of payments technical assistance mission, the CBG has speeded up the process of compiling balance of payments statistics according to the Balance of Payments Manual, $5^{\text {th }}$ edition (BPM5). The authorities submitted the preliminary data for 2003-05 in October 2006, which were published in the November 2006 issue of International Financial Statistics for the first time. With assistance from the U.K. Department for International Development, the CBG conducted an enterprise survey in March 2006 to collect data for the International Investment Position. In April 2006, the CBG also initiated a survey funded by the World Bank to collect data on selected components of the current account.

The government, with the assistance of the World Bank, recently installed the new version of the Commonwealth Secretariat's debt-reporting and management system.

\section{Publication}

The CBG has begun to regularly disseminate some macroeconomic statistics on its website; until 2005, macroeconomic data were provided to the public only through references in the annual budget speech. The CBG has also resumed the publication of its annual reports and quarterly bulletins by posting in August 2006 its annual reports for 2003 and 2004 as well as several quarterly bulletins for 2004 and 2005. The CSD/GBOS's publications are not issued on a regular basis.

There has been no reporting of data for publication in the Government Finance Statistics Yearbook since 1993 or in the International Financial Statistics (IFS), other than monetary and CPI data, since 1997. As reported above, the authorities have just reported balance of payments statistics for 2003-05 for publication in Balance of Payments Statistics Yearbook and in International Financial Statistics. 
16

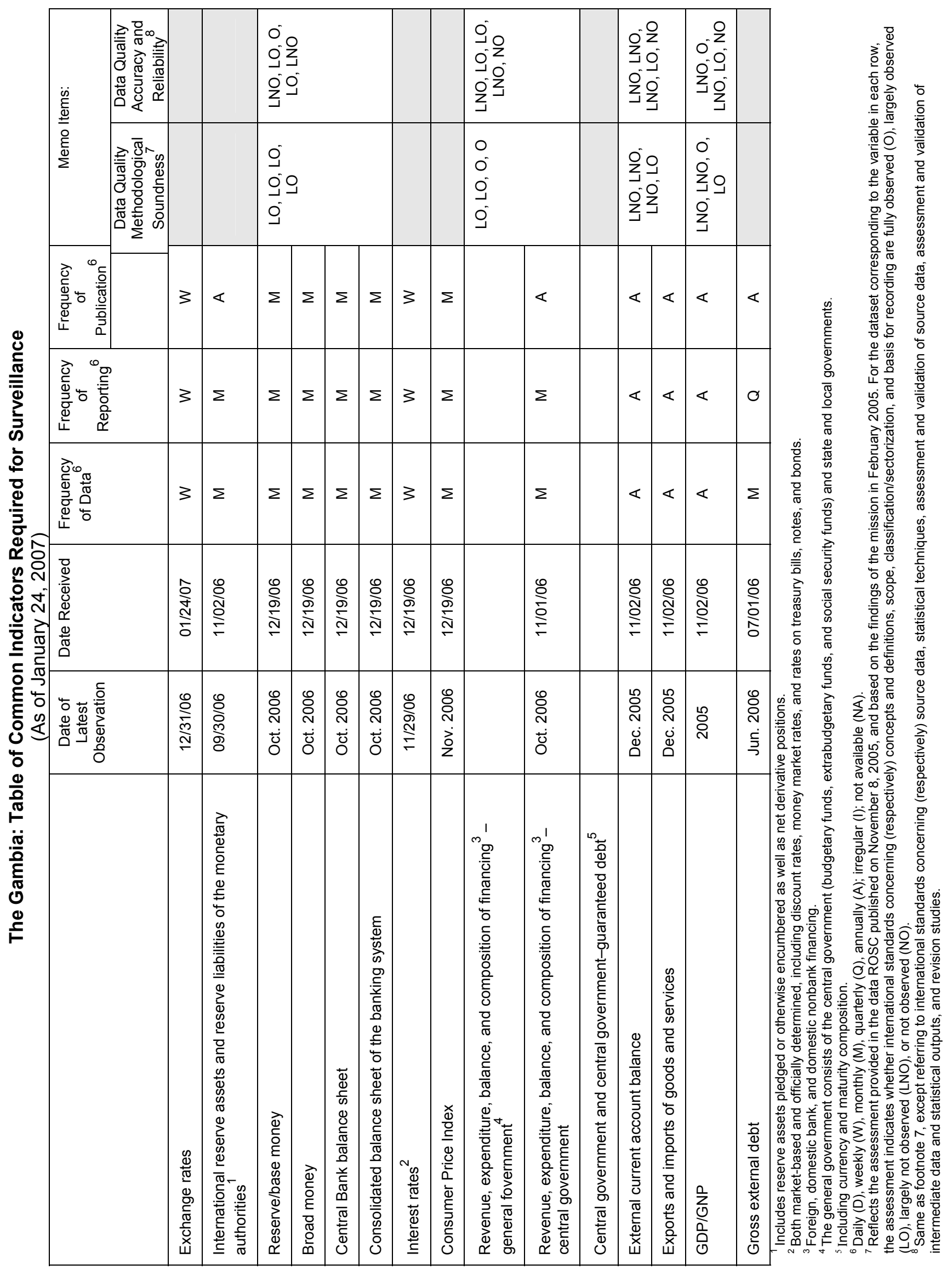


Press Release No. 07/28

International Monetary Fund

FOR IMMEDIATE RELEASE

Washington, D.C. 20431 USA

February 21, 2007

\section{IMF Executive Board Approves US\$21 Million PRGF Arrangement for The Gambia and Additional Interim Assistance under the HIPC Initiative}

The Executive Board of the International Monetary Fund (IMF) today approved a three-year arrangement under the Poverty Reduction and Growth Facility (PRGF) ${ }^{1}$ for The Gambia in an amount equivalent to SDR 14 million (about US\$21 million and 45 percent of quota) in support of the government's economic program into 2009. The first disbursement under the PRGF arrangement will be in an amount equivalent to SDR 2 million (about US\$3 million).

In addition, the Board granted additional interim assistance under the enhanced Heavily Indebted Poor Countries (HIPC) Initiative in an amount equivalent to SDR 0.36 million (about US\$0.5 million).

Following the Executive Board's discussion on The Gambia, Mr. Murilo Portugal, Deputy Managing Director and Acting Chairman, stated:

"The Gambian authorities are to be commended for successfully stabilizing the economy over the last three years. A tightening in monetary policy helped to stabilize the exchange rate and to lower the annual rate of inflation to single-digit levels. Prudent macroeconomic policies and increased external financing helped real GDP growth recover from a droughtinduced decline in 2002 to average about 6 percent annually during 2003-06.

"Notwithstanding the recent achievements, The Gambia continues to face formidable economic challenges including widespread poverty, vulnerability to exogenous shocks, and a heavy public debt burden. The program supported by the new three-year arrangement has

\footnotetext{
${ }^{1}$ The PRGF is the IMF's concessional facility for low-income countries. It is intended that PRGFsupported programs are based on country-owned poverty reduction strategies adopted in a participatory process involving civil society and development partners and articulated in a Poverty Reduction Strategy Paper (PRSP). This is intended to ensure that PRGF-supported programs are consistent with a comprehensive framework for macroeconomic, structural, and social policies to foster growth and reduce poverty. PRGF loans carry an annual interest rate of 0.5 percent and are repayable over 10 years with a 5 1/2-year grace period on principal payments.
} 
been designed to consolidate macroeconomic stabilization while also addressing the above challenges. It is based on the authorities' recently completed second Poverty Reduction Strategy Paper that integrates the Millennium Development Goals into its objectives.

"Maintaining fiscal discipline will be critical to the achievement of the program's mediumterm macroeconomic objectives. In particular, containing the government's domestic borrowing requirement is needed to help lower real interest rates from their current very high levels, and thus stimulate private investment as well as create fiscal space for increasing priority government expenditures.

"The authorities are taking steps to strengthen public financial management and accountability. The launching of the Integrated Financial Management Information System in January 2007 is a key step in improving budget execution and monitoring, and should facilitate the establishment of an effective commitment control system and better alignment of budget execution with PRSP priorities.

"The Central Bank of The Gambia (CBG) deserves commendation for enhancing its monetary operations and for the steps it has taken to address the breakdown in internal controls that led to monetary policy lapses in the early 2000s. The program includes measures to tackle remaining weaknesses and vulnerabilities, and to strengthen the operational independence of the CBG.

"The proposed policies and external debt relief are expected to help The Gambia achieve debt sustainability. Satisfactory implementation of the PRGF-supported program for at least six months is one of the pre-requisites for The Gambia to reach completion point under the enhanced initiative for Heavily Indebted Poor Countries and to become eligible for assistance under the Multilateral Debt Relief Initiative. The authorities have indicated their commitment to fully implement the program," Mr. Portugal said. 


\section{Background}

ANNEX

The Gambia's macroeconomic performance has improved significantly over the last few years. Real GDP growth recovered from a drought-induced decline in 2002 to average about 6 percent annually during 2003-2006, outpacing estimated 2.8 percent annual population growth. The fastest growing sectors were hotels and restaurants (reflecting increased tourist arrivals), construction, and telecommunications. The authorities also succeeded in reducing inflation from a peak 21 percent per year in August 2003 to annual rates below 3 percent since June 2005. Fiscal performance in 2006 was stronger than the previous year, but not as strong as envisaged in the budget on account of expenditure overruns associated with an African Union summit held in Banjul in July. The economy has stayed relatively competitive in spite of a slight appreciation of the nominal and real exchange rates.

\section{Program Summary}

The primary objectives of the program are to consolidate recent macroeconomic achievements and promote sustained high growth and poverty reduction. The program draws on the authorities' recently completed second Poverty Reduction Strategy Paper (PRSP II), which integrates the Millennium Development Goals (MDGs) into its objectives. It targets annual growth of 6-7 percent between 2007 and 2009, annual inflation in the range of 2-4 percent, and fiscal basic balance surpluses of about 3 percent of GDP a year to reduce domestic public debt to a sustainable path. Based on indications from donors, the program projects a significant increase in net external financing which will allow for increased growth-promoting and poverty-reducing government spending. Structural reforms include measures to enhance internal controls and operational independence of the central bank to underpin macroeconomic stability, strengthen public financial management and accountability to ensure that public resources are used effectively and efficiently, and deepen financial intermediation. 
The Gambia: Selected Economic and Financial Indicators, 2004-11

$\begin{array}{llllllll}2004 & 2005 & 2006 & 2007 & 2008 & 2009 & 2010 & 2011\end{array}$

Est. Proj. Proj. Proj. Proj. Proj.

(Annual percentage changes, unless otherwise indicated)

National income and prices

Nominal GDP (millions of dalasis)

$\begin{array}{llllllll}12,042 & 13,182 & 14,248 & 15,732 & 17,261 & 18,894 & 20,637 & 22,540\end{array}$

Nominal GDP

GDP at constant prices

Consumer price index (period average)

Consumer price index (end of period)

$\begin{array}{rrrrrrrr}20.1 & 9.5 & 8.1 & 10.4 & 9.7 & 9.5 & 9.2 & 9.2 \\ 7.0 & 5.1 & 6.5 & 7.0 & 6.0 & 6.0 & 6.0 & 6.0 \\ 14.2 & 3.2 & 1.5 & 3.2 & 3.5 & 3.2 & 3.0 & 3.0 \\ 8.0 & 1.8 & 2.0 & 3.5 & 3.5 & 3.0 & 3.0 & 3.0 \\ & & & & & & & \\ 10.5 & -5.7 & 31.5 & 3.4 & 6.3 & 1.5 & 10.0 & 4.9 \\ 47.5 & -72.4 & 276.7 & 3.9 & 7.3 & 5.0 & 11.1 & 11.8 \\ 46.2 & 9.8 & 11.8 & 10.8 & 3.7 & 3.0 & 6.5 & 3.1 \\ -10.2 & -14.3 & -7.5 & 2.2 & 3.5 & 3.4 & 3.2 & 1.8 \\ -11.3 & 4.2 & 1.9 & \ldots & \ldots & \ldots & \ldots & \ldots \\ -1.2 & 4.5 & 0.2 & \ldots & \ldots & \ldots & \ldots & \ldots\end{array}$

Money and credit

Broad money

Net foreign assets

Net domestic assets

Credit to the government (net) ${ }^{4}$

Credit to the private sector and public enterprises

Claims on foreign exchange bureaus

Other items net

Velocity (GDP/average broad money)

Yield on treasury bills (percent per year) ${ }^{5}$

(Percent change in beginning-of-year broad money)

$\begin{array}{rrrrrrrr}18.3 & 13.1 & 16.5 & 13.3 & 12.0 & 10.0 & 9.8 & 9.8 \\ 28.8 & 0.3 & 7.4 & 9.9 & 3.9 & 4.0 & 2.8 & 2.9 \\ -10.5 & 12.8 & 9.1 & 3.3 & 8.1 & 6.1 & 7.1 & 6.9 \\ -10.6 & 6.3 & 6.2 & 0.0 & -1.2 & -0.9 & -2.2 & -2.7 \\ -6.5 & 5.5 & 9.1 & 5.9 & 5.9 & 5.7 & 6.0 & 6.1 \\ -1.2 & 0.0 & -0.6 & -0.4 & -0.3 & -0.2 & -0.2 & -0.1 \\ 7.7 & 1.0 & -5.6 & -2.2 & 3.7 & 1.5 & 3.5 & 3.6 \\ 2.4 & 2.3 & 2.1 & 2.1 & 2.0 & 2.0 & 2.0 & 2.0 \\ 28.0 & 12.5 & 10.7 & \ldots & \ldots & \ldots & \ldots & \ldots\end{array}$

Gross domestic investment and savings

Gross investment

Gross domestic savings

Gross national savings

Central government budget

Domestic revenue

Grants

Total expenditure and net lending

Overall balance, including grants

Basic balance $^{6}$

Basic primary balance ${ }^{7}$

Net foreign financing

Net domestic financing

(Percent of GDP)

$\begin{array}{rrrrrrrr}26.7 & 25.7 & 23.1 & 26.2 & 23.1 & 22.4 & 21.3 & 20.2 \\ 0.8 & -2.6 & 1.0 & 3.6 & 2.9 & 3.3 & 3.5 & 3.8 \\ 14.1 & 5.5 & 8.7 & 13.3 & 11.4 & 11.7 & 11.0 & 10.5 \\ & & & & & & & \\ 20.9 & 19.7 & 21.6 & 21.3 & 21.3 & 21.2 & 21.3 & 21.2 \\ 4.5 & 1.7 & 0.8 & 8.6 & 5.0 & 4.5 & 4.2 & 3.8 \\ 31.1 & 30.0 & 27.1 & 28.4 & 27.4 & 26.5 & 25.3 & 24.8 \\ -5.7 & -8.6 & -4.7 & 1.4 & -1.1 & -0.7 & 0.2 & 0.2 \\ 2.4 & -0.1 & 1.3 & 3.1 & 2.4 & 2.6 & 3.2 & 3.0 \\ 9.6 & 8.5 & 7.9 & 8.3 & 6.6 & 5.9 & 6.0 & 5.3 \\ 5.7 & 5.5 & 2.2 & 1.1 & 2.6 & 1.5 & 1.8 & 1.8 \\ 0.5 & 3.7 & 2.4 & -2.5 & -1.5 & -0.8 & -2.0 & -2.0 \\ 32.9 & 35.5 & 31.6 & 30.3 & 26.4 & 23.1 & 19.0 & 15.2\end{array}$

Stock of domestic public debt 
External sector

Current account balance

Excluding official transfers

$\begin{array}{llllllll}-21.2 & -25.3 & -18.3 & -21.9 & -19.3 & -17.7 & -16.8 & -15.6\end{array}$

Including official transfers

$\begin{array}{llllllll}-12.6 & -20.2 & -14.3 & -12.9 & -11.7 & -10.7 & -10.4 & -9.7\end{array}$

(Millions of U.S. dollars, unless otherwise indicated)

Current account balance

\section{Excluding official transfers}

Including official transfers

Overall balance of payments

Gross official reserves

In months of imports, c.i.f.

External public debt

Stock

Stock (percent of GDP)

Net present value of debt (percent of exports) ${ }^{8}$

External debt service (percent of exports) ${ }^{8}$

Use of Fund resources

Purchases/disbursements

Repurchases/repayments

Credit outstanding

$\begin{array}{rrrrrrrr}-84.9 & -116.7 & -92.5 & -121.9 & -117.0 & -116.4 & -119.8 & -121.0 \\ -50.7 & -93.0 & -72.7 & -72.1 & -70.7 & -70.5 & -74.1 & -75.4 \\ 32.9 & 14.6 & 8.9 & 16.1 & -0.6 & 1.1 & -1.6 & 0.2 \\ 84.0 & 96.6 & 101.5 & 109.5 & 120.5 & 133.0 & 139.0 & 143.0 \\ 4.3 & 4.5 & 4.2 & 4.1 & 4.3 & 4.6 & 4.6 & 4.6 \\ & & & & & & & \\ 580.7 & 614.7 & 633.3 & 249.9 & 254.8 & 263.1 & 271.2 & 280.1 \\ 144.8 & 133.2 & 125.0 & 44.9 & 42.1 & 40.0 & 37.9 & 36.0 \\ \ldots & 215.4 & 128.0 & 82.7 & 82.2 & 84.7 & 84.3 & 85.7 \\ 21.0 & 24.0 & 20.8 & 17.2 & 10.1 & 12.9 & 10.1 & 8.9 \\ & & (\text { Millions of SDRs) } & & & \\ 0.0 & 0.0 & 0.0 & 4.0 & 4.0 & 4.0 & 2.0 & 0.0 \\ -7.6 & -1.4 & -2.7 & -11.4 & 0.0 & 0.0 & 0.0 & 0.0 \\ 15.9 & 14.6 & 11.8 & \ldots & \ldots & \ldots & \ldots & \ldots \\ & & & & & & & \end{array}$

Sources: Gambian authorities; and IMF staff estimates and projections.

${ }^{1}$ Computed based on values in U.S. dollars.

${ }^{2}$ Excluding reexports and imports for reexport.

${ }^{3}$ Data for 2006 represent as of October 2006.

${ }^{4}$ Including advances to the government in foreign currencies.

${ }^{5}$ Weighted average for all maturities based on weekly auction data. Data shown for 2006 are for November.

${ }^{6}$ Defined as domestic revenue minus expenditure and net lending, excluding externally financed capital expenditure.

${ }^{7}$ Defined as domestic revenue minus expenditure and net lending, excluding interest payments and externally financed capital expenditure.

${ }^{8}$ Exports of goods and nonfactor services (not including re-exports). 



\section{Statement by Peter Gakunu, Executive Director for The Gambia and Ms. Leonia Lephoto, Advisor to the Executive Director February 21, 2007}

\section{Introduction}

On behalf of our Gambian authorities, we would like to thank staff for the productive discussions during the last consultations and negotiations on the new PRGF arrangement with the Fund. We also thank the Executive Board and Management for their continued support. The authorities value the advice proffered by the Fund and are in general agreement with the thrust of the staff report. The Gambia has made significant progress towards macroeconomic stability in recent years, driven by the strengthened macroeconomic management. However, the economy continues to be vulnerable to external shocks; public debt remains high; and unsustainable and poverty is widespread. In this regard, the authorities are requesting a new PRGF arrangement with the Fund. The new PRGF supported program will form the basis of the Gambia's relationship with the Fund for the next three years and will be instrumental in anchoring the reform program and obtaining debt relief under the enhanced HIPC and MDRI. This relief will create fiscal space which will facilitate allocation of resources to development and poverty reducing activities.

\section{Recent economic developments}

Macroeconomic performance continued to be solid during the past year, with robust and generally broad based growth. The main contributors to growth were the construction, telecommunications, agriculture, and services sectors. Inflation remained in the low singledigits, reflecting the prudent monetary policy stance adopted by the authorities. The dalasi remained stable, supported by the strong economic fundamentals and robust foreign exchange inflows.

Fiscal performance is estimated to have improved in 2006 despite the increased fiscal pressures posed by the Gambia's hosting of the African Union (AU) summit and the presidential elections. Revenue performance was broadly in line with the budget while spending was higher than budgeted as a result of the one-off expenditures on the AU summit. Domestic interest payments fell from 47 percent of current spending in 2005 to about 35 percent at the end of 2006, as a result of lower interest rates.

The external current account deficit (including official transfers) narrowed significantly in 2006, owing to the strong growth in tourism earnings, and the rebound of groundnut exports following their collapse in 2005 on account of a failure in marketing arrangements. The current account deficit continued to be financed by foreign direct investment and official concessional loans. The overall external position remained comfortable, with gross international reserves equivalent to about four months of imports. 


\section{Macroeconomic policies}

The new PRGF supported program is aimed at consolidating macroeconomic stability and fostering conditions conducive to high and sustainable growth as well as poverty reduction. In the medium term (2007-09), the program envisages real GDP growth of 6-7 percent, annual inflation in the range of 2-3 percent and improvement in the fiscal basic balance to a surplus of about 2.7 percent of GDP. The improvement in the fiscal basic balance will be supported by lower interest payments, associated with reduced domestic borrowing by the government. The external current account deficit (including official transfers) is expected to fall from 14 percent of GDP in 2006 to 11 percent of GDP in 2009, supported by strong growth in tourism earnings. International reserves will be maintained at the equivalent of about four months of imports.

\section{Fiscal policy}

Fiscal policy will continue to be the main instrument for consolidating macroeconomic stability. In this vein, the authorities are committed to redouble their efforts to sustain the significant progress achieved in improving fiscal performance in recent years. They will maintain fiscal discipline in order to contain domestic borrowing and reduce the domestic public debt to sustainable levels.

The reduction in domestic debt will ease budgetary pressure imposed by the currently high interest payments, which have tended to crowd out poverty reducing and growth promoting expenditures. The authorities therefore, plan to contain domestic borrowing and to finance the future budget shortfalls through concessional borrowing and grants. On the revenue side, government is implementing measures to boost revenues through efficiency gains from improved tax administration. To this end, the Gambia Revenue Authority has been established and a number of measures are being implemented to broaden the tax base and increase tax compliance.

The authorities are continuously making efforts to improve the budget formulation processes and stem the problem of arrears that arise mainly as a result of inadequate budgetary provisions for counterpart funding of donor funded projects. They have continued to implement measures to strengthen expenditure management, including the launching of the Integrated Financial Management Information System (IFMIS) in January 2007. The IFMIS is expected to enhance all the accounting aspects of the budget process, including commitment control and generation of reports for budget monitoring.

The authorities are also taking steps to improve transparency, comprehensiveness and timeliness of reporting on fiscal operations. In this regard, they are establishing a central project management and aid coordination directorate unit whose responsibilities will include

compiling comprehensive information on aid flows into the country and the uses to which the resources are put. A reporting framework for collection of data from public enterprises has also been formulated in order to facilitate monitoring of their fiscal risk and improve fiscal transparency. Significant progress has been made to improve accountability in the use of public resources by clearing the backlog of unaudited government accounts. To date, accounts for fiscal years up to 2004 have been submitted to the Auditor General and it is 
expected that by the end of 2007, the submission of annual accounts will have been brought up to date.

To improve the effectiveness and efficiency of the civil service, the authorities have initiated work on a comprehensive civil service reform program. In this regard they have requested the assistance of the World Bank to undertake a study that would guide the formulation and implementation of a plan to establish a more efficient and better remunerated civil service. The African Development Bank is also considering a project targeted at improving the The Gambia's capacity to implement the PRSP.

\section{Monetary policy and financial sector}

Monetary policy continues to focus on the pursuit of price stability. The monetary targeting framework will continue to be used to achieve this objective, building on the recent success in bringing inflation down to low single digits. In order to further enhance the CBG's capacity in execution of monetary policy, the government will continue to strengthen the operational independence of the central bank in line with the provisions of the CBG Act (2005). To this end, the government will recapitalize the CBG to the tune of D100 million over a period of five years, covering 2006-2010. The authorities also plan to bring government borrowing from the central bank in line with the 10 percent of the previous year's tax revenue limit stipulated by the CBG Act (2005) by the end of 2007. A plan on how this will be executed has been jointly developed by the CBG and the government.

The financial sector in The Gambia is sound with banks well capitalized, profitable and having relatively low levels of non performing loans. However, credit extension to the private sector remains low as the banks continue to be risk averse. In an effort to facilitate bank lending, the CBG is establishing a credit bureau which will provide reference on credit worthiness of potential borrowers seeking credit from the financial sector. Enhanced competition in the banking sector as a result of the operations of the newly licensed banks is also expected to facilitate some improvement in financial intermediation as well as the reduction in the currently wide spread between lending and deposit rates. The CBG has also reduced the required reserve ratio for banks thus increasing their scope for lending.

\section{Promotion of private sector-led growth}

The authorities remain committed to the implementation of policy measures that facilitate the attainment of high, sustainable and broad based growth necessary to reduce poverty and meet the country's development goals. In this regard they place great importance to the role of competitiveness and the investment climate in promoting private sector led growth. Regional cooperation and global economic integration are also high on the authorities' development agenda. In this vein the Gambia implemented the ECOWAS Common external tariff (CET) at the beginning of 2006 and increased sales tax in line with regional taxes. While this is likely to have a negative effect on the competitiveness of The Gambia's re-export trade with neighboring countries, the authorities believe that, given the small size of the economy, the potential benefits of regional integration and duty free access to the whole ECOWAS market will surpass the costs. 
As part of the strategy to improve The Gambia's external competitiveness and the investment climate, the authorities have, with the support of the World Bank, implemented the "Gateway" project which aims to make The Gambia a globally competitive export and processing centre through the expansion of private investment in export oriented production and employment. Progress made under the project includes the establishment of the Gambia Investment Promotion and Free Zone Agency (GIPFZA), implementation of several road projects to improve transportation infrastructure, development of physical infrastructure for the free zone and opening up of the energy and telecommunications sectors to private participation.

The authorities are also reviewing the investment legislation and incentives regime in order to further improve the investment climate. The World Bank is currently assisting with a number of studies that will provide input into the formulation of the country's action plan.

\section{HIPC completion point}

The Gambia had reached HIPC decision point in December 2000 but the authorities were not able to implement a program that could have helped them build the necessary track record for advancing towards HIPC completion point. However, the satisfactory performance for at least six months under the new PRGF supported program will, together with the fulfillment of other HIPC completion point triggers, facilitate reaching completion point and make the country eligible for debt relief under both HIPC and MDRI. This is very important for The Gambia as the level of public debt is very high and unsustainable and without full delivery of this debt relief, The Gambia is deemed to be under debt distress. The authorities are fully committed to the successful implementation of the program. Significant progress towards meeting completion point triggers has also been made and it is hoped that the completion point will be reached by mid 2007 . The fiscal space provided by the debt relief will be used to increase pro-poor spending.

\section{Prior actions to the program}

The authorities and staff had agreed on a set of three prior actions to be implemented before the Board consideration of their PRGF request. We wish to inform the board that these have been successfully implemented. The National Assembly has approved the 2007 Budget which aims at a basic fiscal balance that is in line with the program; the 2003 government accounts have been submitted to the Auditor General for audit; and the action plan for aligning government borrowing with the CBG Act (2005) by the end of 2007 has been formulated. 


\section{Conclusion}

In conclusion, we wish to reaffirm the authorities' commitment to the successful implementation of the program, which underpins consolidating progress made towards macroeconomic stability, promotion of growth, and implementation of a structural reform agenda. They are confident that, with the continued support of the Fund and the international community, they will deliver on the program and propel The Gambia into a higher and more sustainable growth path. 